\title{
SHAPE DERIVATIVE OF DRAG FUNCTIONAL
}

\author{
P. I. PLOTNIKOV AND J. SOKOLOWSKI
}

\begin{abstract}
In the paper compressible, stationary Navier-Stokes (N-S) equations are considered. The model is well-posed, there exist weak solutions in bounded domains, subject to inhomogeneous boundary conditions. The shape sensitivity analysis is performed for N-S boundary value problems, in the framework of small perturbations of the so-called approximate solutions. The approximate solutions are determined from Stokes problem and the small perturbations are given by solutions to the full nonlinear model. Such solutions are unique. The differentiability of the specific solutions with respect to the coefficients of differential operators implies the shape differentiability of the drag functional. The shape gradient of the drag functional is derived in the classical and useful for computations form, an appropriate adjoint state is introduced to this end. The shape derivatives of solutions to the Navier-Stokes equations are given by smooth functions, however the shape differentiability can be shown only in a weak norm. The proposed method of shape sensitivity analysis is general. The differentiability of solutions to the Navier-Stokes equations with respect to the data leads to the first order necessary conditions for a broad class of optimization problems. The boundary shape gradient as well as the boundary value problems for the shape derivatives of solutions to state equations and the adjoint state equations are obtained in the form of singular limits of volume integrals. This method of shape sensitivity analysis seems to be new and appropriate for nonlinear problems. It is an important observation for the numerical methods of shape optimization in fluid mechanics.
\end{abstract}

\section{INTRODUCTION}

In the present paper we derive the boundary form of the shape gradient for the drag functional. In [20] the weak material derivatives are employed to obtain the shape derivative of the drag functional for the compressible Navier-Stokes equations. It is shown in this way that the drag functional is shape differentiable in the sense of [26]. For the smooth obstacle, by the so-called Hadamard representation formula it follows that the shape gradient of the drag is given by the distribution supported on the boundary of the obstacle. In such a formula, only the deformations of the obstacle in the normal direction are present by the Hadamard formula. We use the result and identify the density of the shape gradient by a direct approach. This approach is interesting on its own, it furnishes a new method for the shape analysis for nonlinear problems.

We introduce our method for a simple model problem, replacing the compressible Navier-Stokes boundary value problem by a nonlinear elliptic equation.

2000 Mathematics Subject Classification. Primary: 76N10; 35Q30; 76N25;

Key words and phrases. Compressible Navier-Stokes equations, drag minimization, shape derivative, necessary optimality conditions. 
Example. Let us consider the bounded domain $\Omega$ with the smooth boundary. Take the state equation in the form

$$
-\Delta u(x)=F(x, u(x)) \quad \text { in } \quad \Omega, \quad u(x)=u_{0} \equiv \text { const. } \quad \text { on } \quad \partial \Omega \text {. }
$$

The shape optimization problem is an optimal choice of the domain within an admissible class, in such a way that the boundary shape functional defined on $\partial \Omega$ is minimized. The functional is selected in the form which looks like the $d r a g$, i.e.,

$$
j(\partial \Omega)=u_{0} \int_{\partial \Omega} \nabla u(x) \cdot n(x) d S
$$

Using the Gauss formula we can rewrite this functional in the distributed form

$$
j(\partial \Omega) \equiv \int_{\Omega}\left(|\nabla u|^{2}-u F(u, x)\right) d x
$$

Representation of such kind are common in viscous fluid dynamics since they have clear physical meaning. For instance, the gradient parts represents the rate of dissipation of the energy. However functional (1.1) is only weakly lower semicontinuous in the energy space and can be used mostly for minimization problems. The other approach employed in the paper can be describe in the following way. Introduce a smooth scalar function $\eta(x)$ such that $\eta(x) \equiv 1$ on $\partial \Omega$ and rewrite the expression for $j(\partial \Omega)$ in the equivalent form

$$
J(\Omega)=u_{0} \int_{\Omega}(\nabla \eta \cdot \nabla u-\eta F(x, u)) d x .
$$

This functional is weakly continuous, and its principle part is linear with respect to state variable $u$. The technical difficulty is that the integrand contains an arbitrary function $\eta$ while the result is independent of $\eta$, and we have to eliminate the influence of $\eta$ on the results of calculations. For the latter shape functional we perform the shape sensitivity analysis using the material derivatives, therefore in the fixed domain setting. Given the shape derivative of $J(\Omega)$ supported everywhere in $\bar{\Omega}$ we perform the limit passage with the perturbations fields and evaluate the singular limits of all integrals, formally the perturbations are supported on $\partial \Omega$ for such limits. In this way the shape gradient of $j(\partial \Omega)$ supported on the boundary is identified.

It seems that the proposed method of shape sensitivity analysis of the drag functional is new, and the simplest possible in the case of the drag functional dependent on solutions to compressible Navier-Stokes equations.

Drag functional. We assume that the viscous gas occupies the double-connected domain $\Omega=B \backslash \mathfrak{S}$, where $B \subset \mathbb{R}^{3}$, is a hold-all domain with the smooth boundary $\Sigma=\partial B$, and $\mathfrak{S} \subset B$ is a compact obstacle. The boundary of the obstacle is denoted by $\mathcal{S}:=\partial \mathfrak{S}$, for simplicity.

Furthermore, we assume that the velocity of the gas coincides with a constant given vector field $\mathbf{U}$ on the surface $\Sigma$. The state variables: the velocity field $\mathbf{u}$ and the gas density $\varrho$ satisfying the following equations along with the boundary 
conditions

$$
\begin{gathered}
\Delta \mathbf{u}+\lambda \nabla \operatorname{div} \mathbf{u}=R \varrho \mathbf{u} \cdot \nabla \mathbf{u}+\frac{R}{\epsilon^{2}} \nabla p(\varrho) \text { in } \Omega, \\
\operatorname{div}(\varrho \mathbf{u})=0 \text { in } \Omega, \\
\mathbf{u}=\mathbf{U} \text { on } \Sigma, \quad \mathbf{u}=0 \text { on } \mathcal{S}, \\
\varrho=\varrho_{0} \text { on } \Sigma_{\text {in }},
\end{gathered}
$$

where the pressure $p=p(\varrho)$ is a smooth, strictly monotone function of the density, $\epsilon$ is the Mach number, $R$ is the Reynolds number, $\lambda$ is the viscosity ratio, $\varrho_{0}$ is a positive constant, the inlet $\Sigma_{\text {in }}$ and the outlet $\Sigma_{\text {out }}$ are defined by

$$
\Sigma_{\text {in }}=\{x \in \Sigma: \mathbf{U} \cdot \mathbf{n}<0\}, \quad \Sigma_{\text {out }}=\{x \in \Sigma: \mathbf{U} \cdot \mathbf{n}>0\},
$$

respectively. Here $\mathbf{n}$ stands for the outward normal to $\partial \Omega=\Sigma \cup \mathcal{S}$. Boundary value problem (1.2) can be regarded as mathematical model of viscous gas flow around an airfoil $\mathfrak{S}$ tested in wind tunnel. In our denotation the stress tensor is equal to

$$
\mathbb{T}=: \nabla \mathbf{u}+\nabla \mathbf{u}^{*}+(\lambda-1) \operatorname{div} \mathbf{u} \mathbf{I}-\frac{R}{\epsilon^{2}} p \mathbf{I},
$$

and the hydrodynamical force acting on the element $d s$ of the obstacle boundary $\mathcal{S}$ is $-\mathbb{T} \mathbf{n} d s$. Hence the hydro-dynamical force acting on the body $\mathfrak{S}$ is equal

$$
\mathbf{J}(\mathfrak{S})=:-\int_{\mathcal{S}} \mathbb{T} \mathbf{n} d s=-\int_{\mathcal{S}}\left(\nabla \mathbf{u}+(\nabla \mathbf{u})^{*}+(\lambda-1) \operatorname{div} \mathbf{u I}-\frac{R}{\epsilon^{2}} p \mathbf{I}\right) \cdot \mathbf{n} d s .
$$

Note that this expression can be identically rewritten in the form of the volume integral. To this end we fix an arbitrary function $\eta \in C^{\infty}(\Omega)$ such that $\eta=1$ in an open neighborhood of the obstacle $\mathfrak{S}$ and $\eta=0$ in a vicinity of $\Sigma$. Using the identities

$$
\int_{\mathcal{S}} \mathbb{T} \mathbf{n} d s=\int_{\Omega}(\eta \operatorname{div} \mathbb{T}+\mathbb{T} \nabla \eta) d x, \quad \operatorname{div} \mathbb{T}=R \varrho \mathbf{u} \nabla \mathbf{u}
$$

we obtain

$$
(1.3) \mathbf{J}(\mathfrak{S})=:-\int_{\Omega}\left(\nabla \mathbf{u}+(\nabla \mathbf{u})^{*}+(\lambda-1) \operatorname{div} \mathbf{u I}-\frac{R}{\epsilon^{2}} p \mathbf{I}\right) \nabla \eta d x-R \int_{\Omega} \eta \varrho \mathbf{u} \nabla \mathbf{u} d x
$$

The value of $\mathbf{J}$ is independent of the choice of the function $\eta$. The $\operatorname{drag} J_{D}$ is a work in unit time developed by the component of $\mathbf{J}$ parallel to the airfoil speed $\mathbf{U}$,

$$
J_{D}(\mathfrak{S})=\mathbf{U} \cdot \mathbf{J}(\mathfrak{S}) \text {. }
$$

The minimization of the drag is an important problem of applied aerodynamics. From the mathematical point of view the drag optimization problem is the shape optimization problem for the compressible Navier-Stokes equations. The existence and compactness properties of solutions to drag minimization problem were obtained in [9], [10] for non-stationary case and in [19] for stationary problem by using direct methods of calculus of variations. For incompressible Navier-Stokes equations, the existence of nontrivial shape derivatives of solutions and the formula for the shape derivative of the drag functional and adjoint state were obtained in [3], [4] and [22], see also [23] and [24] for some generalizations. For general theory of optimization and control problems for incompressible Navier-Stokes equations see [2] and [11]. The growing literature on numerical and applied aspects of the problem is nicely surveyed in [12] and [15]. For compressible Navier-Stokes equations (1.2), 
the the formula for the shape derivative of the drag functional and adjoint state were derived in [20] under the assumptions that the Reynolds and Mach numbers are sufficiently small. The goal of this paper is to simplify the results of this work and give an efficient representation for the adjoint state as well as of the shape gradient of the drag functional.

In order to formulate the framework for the shape sensitivity analysis we choose the vector field $\mathbf{T} \in C^{2}\left(\mathbb{R}^{3}\right)^{3}$ vanishing in the vicinity of $\Sigma$, and define the mapping

$$
y=x+\varepsilon \mathbf{T}(x)
$$

which describes the perturbation of the shape of the obstacle. For small $\varepsilon$, the mapping $x \rightarrow y$ takes diffeomorphically the flow region $\Omega$ onto $\Omega_{\varepsilon}=B \backslash \mathfrak{S}_{\varepsilon}$, where the perturbed obstacle $\mathfrak{S}_{\varepsilon}=y(\mathfrak{S})$. Let $\left(\overline{\mathbf{u}}_{\varepsilon}, \bar{\varrho}_{\varepsilon}\right)$ be a solution to problem (1.2) in $\Omega_{\varepsilon}$. After substituting $\left(\overline{\mathbf{u}}_{\varepsilon}, \bar{\varrho}_{\varepsilon}\right)$ into the formula for $\mathbf{J}\left(\mathfrak{S}_{\varepsilon}\right)$, the drag becomes the function of the parameter $\varepsilon$.

It is convenient to reduce such an analysis to the analysis of dependence of solutions with respect to the coefficients of the governing equations. To this end, we introduce the functions $\mathbf{u}_{\varepsilon}(x)$ and $\varrho_{\varepsilon}(x)$ defined in the unperturbed domain $\Omega$ by the formulas

$$
\mathbf{u}_{\varepsilon}(x)=\mathbf{N} \overline{\mathbf{u}}_{\varepsilon}(x+\varepsilon \mathbf{T}(x)), \quad \varrho_{\varepsilon}(x)=\bar{\varrho}_{\varepsilon}(x+\varepsilon \mathbf{T}(x)),
$$

where

$$
\mathbf{N}(x)=\operatorname{det}\left(\mathbf{I}+\varepsilon \mathbf{T}^{\prime}(x)\right)\left(\mathbf{I}+\varepsilon \mathbf{T}^{\prime}(x)\right)^{-1} .
$$

is the adjugate matrix of the Jacobi matrix $\mathbf{I}+\varepsilon \mathbf{T}^{\prime}$. Furthermore, we also use the notation $\mathfrak{g}(x)=\sqrt{\operatorname{det} \mathbf{N}}$. Calculations show [20], that for $\mathbf{u}_{\varepsilon}, \varrho_{\varepsilon}$, the following boundary value problem is obtained,

$$
\begin{gathered}
\Delta \mathbf{u}_{\varepsilon}+\nabla\left(\lambda \mathfrak{g}^{-1} \operatorname{div} \mathbf{u}_{\varepsilon}-\frac{R}{\epsilon^{2}} p\left(\varrho_{\varepsilon}\right)\right)=\mathscr{A}\left(\mathbf{u}_{\varepsilon}\right)+R \mathscr{B}\left(\varrho_{\varepsilon}, \mathbf{u}_{\varepsilon}, \mathbf{u}_{\varepsilon}\right) \text { in } \Omega \\
\operatorname{div}\left(\varrho_{\varepsilon} \mathbf{u}_{\varepsilon}\right)=0 \text { in } \Omega \\
\mathbf{u}_{\varepsilon}=\mathbf{U} \text { on } \Sigma, \quad \mathbf{u}_{\varepsilon}=0 \text { on } \mathcal{S} \\
\varrho_{\varepsilon}=\varrho_{0} \text { on } \Sigma_{\text {in }} .
\end{gathered}
$$

Here, the linear operator $\mathscr{A}$ and the nonlinear mapping $\mathscr{B}$ are defined in terms of $\mathbf{N}$,

$$
\begin{array}{r}
\mathscr{A}(\mathbf{u})=\Delta \mathbf{u}-\left(\mathbf{N}^{*}\right)^{-1} \operatorname{div}\left(\mathfrak{g}^{-1} \mathbf{N} \mathbf{N}^{*} \nabla\left(\mathbf{N}^{-1} \mathbf{u}\right)\right), \\
\mathscr{B}(\varrho, \mathbf{u}, \mathbf{w})=\varrho\left(\mathbf{N}^{*}\right)^{-1}\left(\mathbf{u} \nabla\left(\mathbf{N}^{-1} \mathbf{w}\right)\right) .
\end{array}
$$

In the new variables the expression for the force $\mathbf{J}$ reads

$$
\begin{aligned}
& \mathbf{J}=-\int_{\Omega}\left[\mathfrak{g}^{-1}\left(\mathbf{N}^{*} \nabla\left(\mathbf{N}^{-1} \mathbf{u}_{\varepsilon}\right)+\nabla\left(\mathbf{N}^{-1} \mathbf{u}_{\varepsilon}\right)^{*} \mathbf{N}-\operatorname{div} \mathbf{u}_{\varepsilon}\right)-q_{\varepsilon} \mathbf{I}\right] \mathbf{N}^{*} \nabla \eta d x- \\
& R \int_{\Omega} \varrho_{\varepsilon} \mathbf{u}_{\varepsilon} \nabla\left(\mathbf{N}^{-1} \mathbf{u}_{\varepsilon}\right) \eta d x,
\end{aligned}
$$

where the effective viscous pressure $q$ is given by

$$
q_{\varepsilon}=\frac{R}{\epsilon^{2}} p\left(\varrho_{\varepsilon}\right)-\lambda \mathfrak{g}^{-1} \operatorname{div} \mathbf{u}_{\varepsilon}
$$


In the proposed framework the question of the existence and representation of the derivative $\partial_{\varepsilon} J_{D}\left(\mathfrak{S}_{\varepsilon}\right)$ is reduced to the question on dependence of solutions to the boundary value problem on the coefficients of governing equations. The preference of such an approach is that the equations are considered in fixed domain, which makes the calculations more transparent, but formally more complicated. Moreover, the obtained results depend on the extension of the mapping $\mathbf{T}(x)$ over $\mathbb{R}^{3}$ which leads to the different formulae for different choice of $\mathbf{T}$. It is worthy to note that the shape derivative depends only on restriction of the vector field $\mathbf{T}$ on $\mathcal{S}$. It is independent of the extension of $\mathbf{T}$ over the flow domain. Moreover, expression (1.9) involves an arbitrary function $\eta$ while the shape derivative is independent of $\eta$.

The other approach widely used in the shape optimization is a direct analysis of the problem in variable domain. This leads to the representation of the shape derivative in the form of the integral over $\mathcal{S}$ with the integrand depending only on $\mathbf{T} \cdot \mathbf{n}$ which can be regarded as infinitesimal shift of $\mathcal{S}$. The goal of the paper is to compare both these approaches and to prove that the expression for shape derivatives in the form of surface integral is a singular limit of the derivative of functional (1.9) for the perturbation $\mathbf{T}$ which is concentrated near the boundary. Before formulation of the result we recall the expression for the shape derivative of the drag and the formulation of adjoint state equation.

We follow approach proposed in [18], [20] and restrict our considerations by the case $\lambda \gg 1, R \ll 1$, and $\epsilon \ll 1$ which corresponds to almost incompressible flow with low Reynolds number. In such a case a solution $\left(\mathbf{u}_{0}, q_{0}\right)$ to the Stokes equations

$$
\begin{gathered}
\Delta \mathbf{u}_{0}-\nabla q_{0}=0, \quad \operatorname{div} \mathbf{u}_{0}=0 \text { in } \Omega \\
\mathbf{u}_{0}=\mathbf{U} \text { on } \Sigma, \quad \mathbf{u}_{0}=0 \text { on } \mathcal{S}, \quad \Pi q_{0}=q_{0} \quad \Pi q=: u-\frac{1}{\operatorname{meas} \Omega} \int_{\Omega} q d x .
\end{gathered}
$$

and a constant $\varrho_{0}$ can be regarded as an approximation for a solution to problem (1.7). Instead of the Mach number we introduce the new parameters

$$
\sigma_{0}=R /\left(\lambda \epsilon^{2}\right), \quad \sigma=\sigma_{0} \rho_{0} p^{\prime}\left(\rho_{0}\right) .
$$

Hence we can look for such a solution to this problem in the form

$$
\mathbf{u}_{\varepsilon}=\mathbf{u}_{0}+\mathbf{v}, \quad \varrho_{\varepsilon}=\varrho_{0}+\varphi, \quad q_{\varepsilon}=q_{0}+\lambda \sigma_{0} p\left(\varrho_{0}\right)+\pi+\lambda m,
$$

with the unknowns functions $\vartheta=(\mathbf{v}, \pi, \varphi)$ and the unknown constant $m$. The peculiarity of stationary boundary problem for compressible Navier-Stokes equations is that mass balance equation (1.7b) degenerates at points where $\mathbf{u}_{\varepsilon}$ vanishes. The simple algebraic scheme which allows to cope with this difficulty was proposed in [18]. The basic idea is to consider the effective viscous pressure as new unknown variable, next, to add equality (1.10) to basic system (1.7), and finally, eliminate the divergence $\mathbf{u}_{\varepsilon}$ from mass balance equation (1.7b) using relation (1.10). Thus we come to the following boundary problem for the triplet $(\mathbf{v}, \pi, \varphi)$,

$$
\begin{array}{r}
\Delta \mathbf{v}-\nabla \pi=\mathscr{A}\left(\mathbf{u}_{\varepsilon}\right)+R \mathscr{B}\left(\varrho_{\varepsilon}, \mathbf{u}_{\varepsilon}, \mathbf{u}_{\varepsilon}\right) \text { in } \Omega, \\
\operatorname{div} \mathbf{v}=\mathfrak{g}\left(\frac{\sigma}{\varrho_{0}} \varphi-\Psi-m\right) \text { in } \Omega, \\
\mathbf{u}_{\varepsilon} \cdot \nabla \varphi+\sigma \varphi=\Psi_{1}+m \mathfrak{g} \varrho \text { in } \Omega, \\
\mathbf{v}=0 \text { on } \partial \Omega, \quad \varphi=0 \text { on } \Sigma_{\text {in }}, \quad \Pi \pi=\pi,
\end{array}
$$


where

$$
\begin{gathered}
\Psi_{1}=\mathfrak{g}\left(\varrho \Psi-\frac{\sigma}{\varrho_{0}} \varphi^{2}\right)+\sigma \varphi(1-\mathfrak{g}), \Psi=\frac{q_{0}+\pi}{\lambda}-\frac{\sigma}{p^{\prime}\left(\varrho_{0}\right) \varrho_{0}} H(\varphi), \\
H(\varphi)=p\left(\varrho_{0}+\varphi\right)-p\left(\varrho_{0}\right)-p \prime\left(\varrho_{0}\right) \varphi
\end{gathered}
$$

the vector field $\mathbf{u}_{\varepsilon}$ and the function $\varrho_{\varepsilon}$ are given by (1.12). Next it is necessary to specify the constant $m$. Since the mean value of $\operatorname{div} \mathbf{v}$ is null, the constant $m$ is proportional to the mean value of the quantity $\mathfrak{g}\left(\psi-\varrho_{0}^{-1} \sigma \varphi\right)$. The mean value of the latter quantity involves a large parameter $\sigma$ and it is convenient to replace it by more complicated relation which is independent of the large parameter $\sigma$. Following [20] we can introduce such a condition in the form

$$
m=\varkappa \int_{\Omega}\left(\varrho_{0}^{-1} \Psi_{1} \zeta-\mathfrak{g} \Psi\right) d x, \quad \varkappa=\left(\int_{\Omega} \mathfrak{g}\left(1-\zeta-\varrho_{0}^{-1} \zeta \varphi\right) d x\right)^{-1},
$$

where the auxiliary function $\zeta$ is a solution to the adjoint boundary value problem

$$
-\operatorname{div}\left(\mathbf{u}_{\varepsilon} \zeta\right)+\sigma \zeta=\sigma \mathfrak{g} \text { in } \Omega, \quad \zeta=0 \text { on } \Sigma_{\text {out }},
$$

As it is shown in [20], relations (1.13) define a system of differential equations and boundary conditions which is equivalent to basic problem (1.7). The proposed algebraic scheme works properly in the range of parameters $R \sim \lambda \epsilon^{2}$. The results are available for larger domain in the space of parameters, but in this case the modified equations involve non-local operators, see [16], [17], and [21] for details. The following proposition guarantees the existence and uniqueness of solution to boundary value problem (1.13).

For $s \in(0, \infty), r \in(1, \infty)$, denote by $X^{s, r}$ and $X^{s, r}$ the Banach spaces

$$
X^{s, r}=W^{s, r}(\Omega) \cap W^{1,2}(\Omega), Y^{s, r}=W^{s+1, r}(\Omega) \cap W^{2,2}(\Omega) .
$$

Here $W^{s, r}(\Omega)$ denotes the Sobolev space for an integer $s$ and the fractional SobolevSlobodetski space otherwise. We assume that $\Omega$ satisfies the following condition

Condition 1.1. The flow domain $\Omega$ has a boundary of class $C^{\infty}$ and it admits the representation $\Omega=B \backslash \mathfrak{S}, \mathfrak{S} \Subset B$, in which the hold all domain $B$ with the boundary $\Sigma=\partial B$ is strictly convex.

Proposition 1.2. Let $\mathbf{U}$ be a given vector field and the exponents $s, r$ satisfy the inequalities

$$
1 / 2<s<1, \quad 1<r<3 /(2 s-1), \quad s r>3 .
$$

Then there is $\sigma^{*}$, depending only on $s, r, \Omega$, and $\mathbf{U}$, with the following property. If $\sigma>\sigma^{*}$, then there exist $\mu_{0}>0$ and $\varepsilon_{0}>0$, depending only on $\sigma, s, r, \Omega$, and $\mathbf{U}$, such that for all $\mu \in\left(0, \mu_{0}\right), \varepsilon \in\left[0, \varepsilon_{0}\right]$, and

$$
R+\lambda^{-1}<\mu
$$

problem (1.13) has a solution $(\mathbf{v}, \varphi, \zeta, m) \in Y^{s, r} \times\left(X^{s, r}\right)^{3} \times \mathbb{R}$. Moreover, this solution admits the estimates

$$
\left\|\mathbf{u}_{\varepsilon}\right\|_{Y^{s, r}}+\|\zeta\|_{X^{s, r}} \leq c_{0}, \quad|m|+\|\varphi\|_{X^{s, r}} \leq c_{0} \mu
$$

where the constant $c_{0}$ is independent of $\mu$ and $\varepsilon$. 
Note that by virtue of $(2.3),(\mathbf{v}, \varphi, \zeta) \in C^{1+\gamma}(\Omega) \times C^{\gamma}(\Omega)^{2}$ with $\gamma(r, s)>0$ and the drag functional $J_{D}(\mathfrak{S})$ is well defined. Recalling the expressions for $\mathscr{A}, \mathscr{B}$, and $\mathfrak{g}$ we conclude that the coefficients of equations (1.13) are analytic functions of the parameter $\varepsilon$. Formal differentiation of both sides of (1.13) leads to the following equations for the variations

$$
\begin{aligned}
& \quad(\delta \mathbf{v}, \delta \pi, \delta \varphi, \delta \zeta, \delta m)=\left.\frac{d}{d \varepsilon}(\mathbf{v}, \pi, \varphi, \zeta, m)\right|_{\varepsilon=0}, \\
& \Delta \delta \mathbf{v}-\nabla \delta \pi=\mathscr{C}(\delta \varphi, \delta \mathbf{v})+\mathscr{D}(\mathbf{D}), \\
& \operatorname{div} \delta \mathbf{v}=b_{21} \delta \varphi+b_{22} \delta \pi+b_{23} \delta m+b_{20} \operatorname{Tr} \mathbf{T}^{\prime}, \\
& \mathbf{u} \nabla \delta \varphi+\sigma \delta \varphi=-\delta \mathbf{v} \nabla \varphi+b_{11} \delta \varphi+b_{12} \delta \pi+b_{13} \delta m+b_{10} \operatorname{Tr} \mathbf{T}^{\prime}, \\
& -\operatorname{div}(\delta \zeta \mathbf{u})+\sigma \delta \zeta=\operatorname{div}(\zeta \delta \mathbf{v})+\sigma \operatorname{Tr} \mathbf{T}^{\prime}, \\
& \delta m=\varkappa \int_{\Omega}\left(b_{31} \delta \varphi+b_{32} \delta \pi+b_{34} \delta \zeta+b_{30} \operatorname{Tr} \mathbf{T}^{\prime}\right) d x, \\
& (\mathbf{I}-\Pi) \delta \pi=0,
\end{aligned}
$$

where the matrix $\mathbf{D}$ and the linear forms $\mathscr{C}, \mathscr{D}$ are given by the equalities

$$
\begin{aligned}
& \mathbf{D}=\left.\frac{d \mathbf{N}}{d \varepsilon}\right|_{\varepsilon=0}=\left(\operatorname{Tr} \mathbf{T}^{\prime}\right) \mathbf{I}-\mathbf{T}^{\prime},\left.\frac{d \mathfrak{g}}{d \varepsilon}\right|_{\varepsilon=0}=\operatorname{Tr} \mathbf{T}^{\prime} \equiv \operatorname{div} \mathbf{T}, \\
& \mathscr{C}(\delta \varphi, \delta \mathbf{v})=R \delta \varphi \mathbf{u} \nabla \mathbf{u}+R \varrho \mathbf{u} \nabla \delta \mathbf{v}+R \varrho \delta \mathbf{v} \nabla \mathbf{u}, \\
& \mathscr{D}(\mathbf{D})=\operatorname{div}\left[\left(\left(\operatorname{Tr} \mathbf{T}^{\prime}\right) \mathbf{I}-\mathbf{D}-\mathbf{D}^{*}\right) \nabla \mathbf{u}\right]+\mathbf{D}^{*} \Delta \mathbf{u}+\Delta(\mathbf{D u})- \\
& R \varrho \mathbf{D}^{*}(\mathbf{u} \nabla \mathbf{u})-R \varrho \mathbf{u} \nabla(\mathbf{D u}),
\end{aligned}
$$

respectively and the coefficients $b_{i j}$ are given by the formulae

$$
\begin{aligned}
& b_{11}=\Psi+m-\frac{2 \sigma}{\varrho_{0}} \varphi-\frac{\varrho \sigma}{p^{\prime}\left(\varrho_{0}\right) \varrho_{0}} H^{\prime}(\varphi), \quad b_{12}=\frac{\varrho}{\lambda}, \\
& b_{13}=\varrho, \quad b_{10}=\varrho(\Psi+m)-\frac{\sigma}{\varrho_{0}} \varphi^{2}-\sigma \varphi, \\
& b_{21}=\frac{\sigma}{\varrho_{0}}+\frac{\sigma}{p^{\prime}\left(\varrho_{0}\right) \varrho_{0}} H^{\prime}(\varphi), \quad b_{22}=-\frac{1}{\lambda}, \quad b_{23}=-1, \quad b_{20}=\frac{\sigma}{\varrho_{0}} \varphi-\Psi-m, \\
& b_{31}=\frac{\zeta}{\varrho_{0}}\left((\Psi+m)-\frac{2 \sigma}{\varrho_{0}} \varphi\right)+\frac{\sigma}{p^{\prime}\left(\varrho_{0}\right) \varrho_{0}}\left(1-\frac{\varrho \zeta}{\varrho_{0}}\right) H^{\prime}(\varphi) \\
& b_{32}=\frac{1}{\lambda}\left(\frac{\varrho}{\varrho_{0}} \zeta-1\right), \quad b_{34}=\frac{1}{\varrho_{0}}\left(\varrho(\Psi+m)-\frac{\sigma}{\varrho_{0}} \varphi^{2}\right), \\
& b_{30}=\left(\frac{\varrho}{\varrho_{0}} \zeta-1\right)(\Psi+m)-\frac{\sigma \varrho}{\varrho_{0}^{2}} \varphi \zeta,
\end{aligned}
$$

in which

$$
\mathbf{u}=\left.\mathbf{u}_{\varepsilon}\right|_{\varepsilon=0}, \quad \varrho=\left.\varrho_{\varepsilon}\right|_{\varepsilon=0}
$$

Formal differentiation of equality (1.9) leads to the following expression for the shape derivative of the drag functional

$$
\left.\frac{d}{d \varepsilon} J_{D}\left(\mathfrak{S}_{\varepsilon}\right)\right|_{\varepsilon=0}=L_{e}(\mathbf{T})+L_{u}(\delta \mathbf{v}, \delta \pi, \delta \varphi),
$$


where the linear forms $L_{e}$ and $L_{u}$ are defined by the equalities

$$
\begin{aligned}
& L_{e}(\mathbf{T})=\mathbf{U} \int_{\Omega} \operatorname{Tr} \mathbf{T}^{\prime}\left(\nabla \mathbf{u}+\nabla \mathbf{u}^{*}-\operatorname{div} \mathbf{u I}\right) \nabla \eta d x- \\
& \mathbf{U} \int_{\Omega}\left[\mathbf{D}^{*} \nabla \mathbf{u}+\nabla \mathbf{u}^{*} \mathbf{D}-\nabla(\mathbf{D u})-\nabla(\mathbf{D u})^{*}\right] \nabla \eta d x- \\
& \mathbf{U} \int_{\Omega}\left[\nabla \mathbf{u}+\nabla \mathbf{u}^{*}-\operatorname{div} \mathbf{u}-q \mathbf{I}\right] \mathbf{D}^{*} \nabla \eta d x+ \\
& R \mathbf{U} \int_{\Omega} \varrho \mathbf{u} \nabla(\mathbf{D u}) \eta \cdot d x
\end{aligned}
$$

and

$(1.22) L_{u}(\delta \mathbf{v}, \delta \pi, \delta \varphi)=-\mathbf{U} \int_{\Omega}\left[\left(\nabla \delta \mathbf{v}+\nabla \delta \mathbf{v}^{*}-\operatorname{div} \delta \mathbf{v}-\delta \pi\right) \nabla \eta+R \mathscr{C}(\delta \mathbf{v}, \delta \varphi) \eta\right] d x$.

The expression for the form $L_{u}$ can be identically rewritten in terms of the so-called adjoint state. To this end note that, since $\nabla \eta=0$ on $\partial \Omega$ and div $(\varrho \mathbf{u})=0$, we have

$$
-\mathbf{U} \int_{\Omega}\left[\left(\nabla \delta \mathbf{v}+\nabla \delta \mathbf{v}^{*}-\operatorname{div} \delta \mathbf{v}\right] \nabla \eta d x=\int_{\Omega} \delta \mathbf{v} \cdot \Delta(\eta \mathbf{U}) d x\right.
$$

and

$$
-\mathbf{U} \int_{\Omega} \rho \eta \mathbf{u} \nabla \delta \mathbf{v} d x=\int_{\Omega} \rho(\mathbf{u} \nabla \eta) \mathbf{U} d x
$$

This leads to the integral representation of $L_{u}$ in $L_{2}(\Omega)$ scalar product

$$
L_{u}=\int_{\Omega}(A \cdot \delta \mathbf{v}+B \delta \pi+C \delta \varphi) d x
$$

where

$$
\begin{aligned}
& A=\Delta(\eta \mathbf{U})-R \eta \varrho \nabla \mathbf{u} \mathbf{U}+R \varrho(\mathbf{u} \cdot \nabla \eta) \mathbf{U}, \\
& B=\mathbf{U} \cdot \nabla \eta, \quad C=-R \eta(\mathbf{u} \nabla \mathbf{u}) \cdot \mathbf{U} .
\end{aligned}
$$

We define the adjoint state $\left(\mathbf{h}^{*}, g^{*}, \varsigma^{*}, v^{*}, l^{*}\right)$ as a solution of the boundary value problem for the system of differential equations adjoint to system (1.17)

$$
\begin{gathered}
\Delta \mathbf{h}^{*}-\nabla g^{*}-R \varrho \nabla \mathbf{u h} \mathbf{h}^{*}+R \varrho \mathbf{u} \nabla \mathbf{h}^{*}+\varsigma^{*} \nabla \varphi+\zeta \nabla v^{*}=A, \\
\operatorname{div} \mathbf{h}^{*}-\Pi\left(b_{12} \varsigma^{*}+b_{22} g^{*}+\varkappa b_{32} l^{*}\right)=B, \\
-\operatorname{div}\left(\mathbf{u} \varsigma^{*}\right)+\sigma \varsigma^{*}-R(\mathbf{u} \nabla \mathbf{u}) \cdot \mathbf{h}^{*}-b_{21} g^{*}-b_{11} \varsigma^{*}-\varkappa b_{31} l^{*}=C, \\
\mathbf{u} \nabla v^{*}+\sigma v^{*}-\varkappa b_{34} l^{*}=0, \\
l^{*}-\int_{\Omega} b_{13} \varsigma^{*} d x=0 \\
\mathbf{h}^{*}=0 \text { on } \partial \Omega, \quad \varsigma^{*}=0 \text { on } \Sigma_{\text {out }}, \quad v^{*}=0 \text { on } \Sigma_{\text {in }}, \quad g^{*}=\Pi g^{*} .
\end{gathered}
$$

Using the notion of the adjoint state we can rewrite the expression for $L_{u}$ in the form

$$
L_{u} \equiv \int_{\Omega}\left[\operatorname{Tr} \mathbf{T}^{\prime}\left(b_{10} \varsigma^{*}+b_{20} g^{*}+\sigma v^{*}+b_{30} l^{*}\right)+\mathscr{D}(\mathbf{D}) \mathbf{h}^{*}\right] d x,
$$

It is important to note that the adjoint state equations are independent on $\mathbf{T}$, but involve an arbitrary function $\eta$. Notice also that the value of the shape derivative 
depends only on the restriction of $\mathbf{T}$ to $\mathcal{S}$, i.e., only on the perturbation of the surface $\mathcal{S}$. In the generic case the perturbation of $\mathcal{S}$ can be taken as follows

$$
\mathcal{S}_{\varepsilon}=\{x=\omega+\varepsilon f(\omega) \mathbf{n}(\omega), \quad \omega \in \mathcal{S}\},
$$

where the normal shift $f(\omega)$ is a smooth function defined on $\mathcal{S}$. This relation shows that the mapping $\mathbf{T}$ satisfies the boundary condition

$$
\mathbf{T}(\omega)=f(\omega) \mathbf{n}(\omega) \text { for } \omega \in \mathcal{S} .
$$

It is reasonable to eliminate $\eta$ and $\mathbf{T}$ from formulae (1.20) and reformulate the expressions for the forms $L_{e}$ and $L_{u}$, only in terms of the normal shift $f(\omega)$. The corresponding result is given by the following theorem which is the main result of this paper.

Theorem 1.3. Let parameters $R, \lambda, \sigma$, flow domain $\Omega$, and exponents $s, r$ meet all requirements of Proposition 1.2, and the perturbed surface $\mathcal{S}_{\varepsilon}$ is defined by equation (1.27) with $f \in C^{\infty}(\mathcal{S})$, then

$$
\left.\frac{d}{d \varepsilon} J_{D}\left(\mathfrak{S}_{\varepsilon}\right)\right|_{\varepsilon=0}=\int_{\mathcal{S}} f(\omega)\left[b_{10} \varsigma+b_{20}^{0} g+\sigma v+l b_{30}-\left(\partial_{n} \mathbf{h} \cdot \mathbf{n}\right)\left(\partial_{n} \mathbf{u} \cdot \mathbf{n}\right)\right] d s
$$

with the adjoint state variables

$$
(\mathbf{h}, g, \varsigma, v, l) \in W^{1+s, r}(\Omega) \times \Pi W^{s, r}(\Omega) \times W^{s, r}(\Omega)^{2} \times \mathbb{R},
$$

satisfying the following equations and boundary conditions

$$
\begin{gathered}
\Delta \mathbf{h}-\nabla g-R \varrho \nabla \mathbf{u h}+R \varrho \mathbf{u} \nabla \mathbf{h}+\varsigma \nabla \varphi+\zeta \nabla v=0, \\
\operatorname{div} \mathbf{h}-\Pi\left(b_{12} \varsigma+b_{22} g+\varkappa b_{32} l\right)=0, \\
-\operatorname{div}(\mathbf{u} \varsigma)+\sigma \varsigma-R(\mathbf{u} \nabla \mathbf{u}) \cdot \mathbf{h}-b_{21} g-b_{11} \varsigma-\varkappa b_{31} l=0, \\
\mathbf{u} \nabla v+\sigma v-\varkappa b_{34} l=0, \\
l-\int_{\Omega} b_{13} \varsigma d x=0
\end{gathered}
$$

(1.29f) $\mathbf{h}=0$ on $\Sigma, \quad \mathbf{h}=-\mathbf{U}$ on $\mathcal{S}, \quad \varsigma=0$ on $\Sigma_{\text {out }}, \quad v=0$ on $\Sigma_{\text {in }}, \quad g=\Pi g$.

Remark 1.4. It is easily seen that solutions to equations (1.25) and (1.29) are connected by the simple relation

$$
\left(\mathbf{h}^{*}, g^{*}, \varsigma^{*}, v^{*}, l^{*}\right)=(\eta \mathbf{U}, 0,0,0,0)+(\mathbf{h}, g, \varsigma, v, l) .
$$

Hence the dependence of the adjoint state on $\eta$ is additive, while the dependence of $J_{D}$ on $\eta$ is multiplicative.

Remark 1.5. If a solution to problem (1.29) has continuous derivatives, then $\mathbf{u} \nabla \varsigma$, $\mathbf{u} \nabla v$ vanish on $\mathcal{S}$ and equations (1.29c), (1.29d) give simple algebraic relations for boundary values of functions $g$, $\varsigma$, and $v$. Using these relations we can eliminate $\varsigma$ and $v$ from formula (1.28).

It follows from (1.20) that we can split the expression for the shape derivative into the geometrical part $L_{e}$ and dynamical part $L_{u}$. The geometrical part depends only on the shape perturbation and state variables $\mathbf{u}$ and $\varrho$. The calculation of the dynamical part is more complicated and requires the solution of boundary value problem for adjoint state equations. Theorem 1.3 shows that the geometrical part of the shape derivative vanishes and therefore, can not be used in the numerical 
process of the shape optimization in our framework.

The remaining part of the paper is devoted to the proof of Theorem 1.3. In Section 2 we prove the existence of solutions to problems (1.25) and (1.29) with continuously differentiable vector fields $\mathbf{h}^{*}$ and $\mathbf{h}$. In the next section we discuss the properties of the normal coordinates in a neighborhood of $\mathcal{S}$. In Section 4 we derive the formulae for the singular limits of integrals of functions concentrated near $\mathcal{S}$. Finally, in Section 5 the proof of Theorem 1.3 is completed.

\section{Estimates of Solutions to ADJoint State EQuATions}

In this section we prove the well-posedness and derive a priori estimates for solutions of the adjoint state equations.

Function spaces. First we recall some basic facts from the theory of SobolevSlobodetsky spaces, which can be found in [1] and [25]. Let $\Omega$ be the whole space $\mathbb{R}^{d}, d \geq 1$, or a bounded domain in $\mathbb{R}^{d}$ with the boundary $\partial \Omega$ of class $C^{1}$. For an integer $l \geq 0$ and for an exponent $r \in[1, \infty)$, we denote by $W^{l, r}(\Omega)$ the Sobolev space endowed with the norm $\|u\|_{W^{l, r}(\Omega)}=\sup _{|\alpha| \leq l}\left\|\partial^{\alpha} u\right\|_{L^{r}(\Omega)}$. For real $0<s<1$, the fractional Sobolev space $W^{s, r}(\Omega)$ is obtained by the real interpolation method between $L^{r}(\Omega)$ and $W^{1, r}(\Omega)$, and consists of all measurable functions with the finite norm

$$
\|u\|_{W^{s, r}(\Omega)}=\|u\|_{L^{r}(\Omega)}+|u|_{s, r, \Omega}
$$

where

$$
|u|_{s, r, \Omega}^{r}=\int_{\Omega \times \Omega}|x-y|^{-d-r s}|u(x)-u(y)|^{r} d x d y .
$$

In the general case, the Sobolev space $W^{l+s, r}(\Omega)$ is defined as the space of measurable functions with the finite norm $\|u\|_{W^{l+s, r}(\Omega)}=\|u\|_{W^{l, r}(\Omega)}+\sup _{|\alpha|=l}\left\|\partial^{\alpha} u\right\|_{W^{s, r}(\Omega)}$.

Furthermore, the notation $W_{0}^{s, r}(\Omega), 0 \leq s \leq 1$, stands for the closed subspace of $W^{s, r}\left(\mathbb{R}^{d}\right)$ which consists of all functions $u \in W^{s, r}\left(\mathbb{R}^{d}\right)$ vanishing outside of $\Omega$. We will identify functions of $W_{0}^{s, r}(\Omega)$ with their extensions by zero to $\mathbb{R}^{d}$. Recall that $W^{s, r}(\Omega)=W_{0}^{s, r}(\Omega)$ for $s r<1$. It is important to note that $C_{0}^{\infty}(\Omega)$ is dense in $W_{0}^{s, r}(\Omega)$, but the space $W_{0}^{s, r}(\Omega)$ coincides with the completion of $C_{0}^{\infty}(\Omega)$ in the norm of $W^{s, r}(\Omega)$ if and only if $s \neq 1 / r+$ integer.

For all $0<s<1$ and $1<r<\infty$, we denote by $\mathcal{W}_{0}^{s, r}(\Omega)$ the interpolation space $\left[\mathcal{W}_{0}^{0, r}(\Omega), \mathcal{W}_{0}^{1, r}(\Omega)\right]_{s, r}$ endowed with the one of the equivalent norms defined by real interpolation method. In other words, $\mathcal{W}_{0}^{s, r}(\Omega)$ is obtained by real interpolation of the subspace $\mathcal{W}_{0}^{1, r}(\Omega)=W_{0}^{1, r}(\Omega) \subset W^{1, r}(\Omega)$ and $\mathcal{W}_{0}^{0, r}(\Omega)=L^{r}(\Omega)$. Generally speaking, $\mathcal{W}_{0}^{s, r}(\Omega)$ is not a subspace of $W^{s, r}(\Omega)$, and its norm is stronger than the norm of $W^{s, r}(\Omega)$. The question on the interpolation of subspaces is one of the difficult questions of the interpolation theory. In our particular case the following result was obtained in [8] and [13], see also [14] for complete account of the theory,

$$
\mathcal{W}_{0}^{s, r}(\Omega)=W_{0}^{s, r}(\Omega) \text { for } s \neq 1 / r+\text { integer. }
$$

The other application of results [13],[14] is the interpolation of subspaces of finite codimension. Recall denotation (1.11) for the projection $\Pi$. We have for $s \in(0,1)$

$$
\Pi W^{s, r}(\Omega)=\left[\Pi L^{r}(\Omega), \Pi W_{0}^{1, r}(\Omega)\right]_{s, r} .
$$


Embedding theorems. For $s r>d$ and $0 \leq \alpha<s-r / d$, the embedding $W^{s, r}(\Omega) \hookrightarrow$ $C^{\alpha}(\Omega)$ is continuous and compact. Moreover, for $s r>d$, and all $u, v \in W^{s, r}(\Omega)$,

$$
\|u v\|_{W^{s, r}(\Omega)} \leq c(r, s)\|u\|_{W^{s, r}(\Omega)}\|v\|_{W^{s, r}(\Omega)} .
$$

If $s r<d$ and $t^{-1}=r^{-1}-d^{-1} s$, then the embedding $W^{s, r}(\Omega) \hookrightarrow L^{t}(\Omega)$ is continuous, [1] Th. 7.57. We have also, [1] Th.7.58, for $\alpha<s,(s-\alpha) r<d$ and $\beta^{-1}=r^{-1}-d^{-1}(s-\alpha)$,

$$
\|u\|_{W^{\alpha, \beta}(\Omega)} \leq c(r, s, \alpha, \beta, \Omega)\|u\|_{W^{s, r}(\Omega)} .
$$

Duality. Let $s \in[0,1]$. We define

$$
\langle u, v\rangle=\int_{\Omega} u v d x
$$

for all functions such that the right hand side make sense. For $r \in(1, \infty)$, each element $v \in L^{r}(\Omega)$ determines the functional $\mathcal{L}_{v}$ of $\left(W_{0}^{s, r^{\prime}}(\Omega)\right)^{\prime}, r^{\prime}=r /(r-1)$, by the identity $\mathcal{L}_{v}(u)=\langle u, v\rangle$. We introduce the $(-s, r)$-norm of an element $v \in L^{r}(\Omega)$ to be by definition the norm of the functional $\mathcal{L}_{v}$, that is

$$
\|v\|_{W^{-s, r}(\Omega)}=\sup _{\substack{u \in W_{0}^{s, r^{\prime}}(\Omega) \\\|u\|_{W_{0}^{s, r^{\prime}}(\Omega)}=1}}|\langle u, v\rangle| .
$$

Let $W^{-s, r}(\Omega)$ denote the completion of the space $L^{r}(\Omega)$ with respect to $(-s, r)$ norm. The space $W^{-s, r}(\Omega)$ is topologically and algebraically isomorphic to $\left(W_{0}^{s, r^{\prime}}(\Omega)\right)^{\prime}$.

Similarly, we can define the dual space $\mathcal{W}^{-s, r}(\Omega)$ as the completion of $L^{r}(\Omega)$ in the norm

$$
\|v\|_{\mathcal{W}^{-s, r}(\Omega)}=\sup _{\substack{u \in \mathcal{W}_{0}^{s, r^{\prime}}(\Omega) \\\|u\|_{\mathcal{W}_{0}^{s, r^{\prime}}(\Omega)}=1}}|\langle u, v\rangle| .
$$

The space $\mathcal{W}^{-s, r}(\Omega)$ is topologically and algebraically isomorphic to $\left(\mathcal{W}_{0}^{s, r^{\prime}}(\Omega)\right)^{\prime}$. Moreover, we can identify $\mathcal{W}^{-s, r}(\Omega)$ with the interpolation space $\left[L^{r}(\Omega), W_{0}^{-1, r}(\Omega)\right]_{s, r}$, see [5]. Since $s-1 / r^{\prime}$ is not integer for a non-integer $-s-1 / r$, it follows from $(2.2)$ that

$$
\mathcal{W}_{0}^{-s, r}(\Omega)=W_{0}^{-s, r}(\Omega) \text { for }-s \neq 1 / r+\text { integer. }
$$

It is well known that the operator $\nabla: W^{s, r}(\Omega) \mapsto W^{s-1, r}(\Omega)$ is continuous for $s=0,1$. It follows from this that $\nabla: W^{s, r}(\Omega) \mapsto \mathcal{W}^{s-1, r}(\Omega)$ is continuous for all $s \in(0,1)$ and hence

$$
\|\nabla u\|_{W^{s-1, r}(\Omega)} \leq c(s, r)\|u\|_{W^{s, r}(\Omega)} \text { for all } s \neq 1 / r+\text { integer, } s \in[0,1]
$$

The above results leads to the following lemmas which will be used in the proof of the solvability of the adjoint state equations.

Lemma 2.1. Let $\Omega \subset \mathbb{R}^{3}$ be a bounded domain with $\partial \Omega$ of class $C^{2}$, and $(F, G,) \in$ $W^{s-1, r}(\Omega) \times \Pi W^{s, r}(\Omega)$,

$$
s \in[0,1], \quad r \in(1, \infty), \quad s \neq 1 / r+\text { integer. }
$$


Then the boundary value problem

$$
\begin{array}{r}
\Delta \mathbf{v}-\nabla \pi=F, \quad \operatorname{div} \mathbf{v}=\Pi G \quad \text { in } \Omega, \\
\mathbf{v}=0 \quad \text { on } \partial \Omega, \quad \Pi \pi=\pi,
\end{array}
$$

has a unique solution $(\mathbf{v}, \pi) \in W^{s+1, r}(\Omega) \times \Pi W^{s, r}(\Omega)$ such that

$$
\|\mathbf{v}\|_{W^{s+1, r}(\Omega)}+\|\pi\|_{W^{s, r}(\Omega)} \leq c(\Omega, r, s)\left(\|F\|_{W^{s-1, r}(\Omega)}+\|G\|_{W^{s, r}(\Omega)}\right) .
$$

Proof. For $s=0,1$, the lemma is a classical result of the theory of Stokes equations (see [7]). Hence the mapping $(F, G) \rightarrow(\mathbf{v}, \pi)$ defines the continuous operator from $W^{s-1, r} \times \Pi W^{s, r}(\Omega)$ to $W^{s+1, r} \times \Pi W^{s, r}, s=0,1$. By the main theorem of the interpolation theory, this operator is continuous from $\mathcal{W}^{s-1, r} \times$ $\Pi W^{s, r}(\Omega)$ to $W^{s+1, r} \times \Pi W^{s, r}$ for all $s \in(0,1)$. In particular, we have

$$
\|\mathbf{v}\|_{W^{s+1, r}(\Omega)}+\|\pi\|_{W^{s, r}(\Omega)} \leq c(\Omega, r, s)\left(\|F\|_{\mathcal{W}^{s-1, r}(\Omega)}+\|G\|_{W^{s, r}(\Omega)}\right),
$$

which along with (2.9) gives (2.12).

The next lemma gives the multiplicative estimate in fractional Sobolev space. Assume that exponents $s, s^{\prime}$ and $r, r^{\prime}$ satisfy the conditions

$$
s^{\prime}+s=1, \quad \frac{1}{r}+\frac{1}{r^{\prime}}=1,, \quad s, s^{\prime} \in(0,1), \quad r, r^{\prime} \in(1, \infty) .
$$

Lemma 2.2. Let $\Omega \subset \mathbb{R}^{d}$ be a bounded domain with the Lipschitz boundary. Furthermore, assume that exponents $s, s^{\prime}$ and $r, r^{\prime}$ satisfy (2.13) and the conditions

$$
s r>d, \quad 1 / 2<s, \quad s \neq 1 / r+\text { integer. }
$$

Then there is a constant $c$, depending only on $r, s$, and $\Omega$, such that for all $\varsigma \in$ $W^{s, r}(\Omega)$ and $u \in W^{s^{\prime}, r^{\prime}}(\Omega)$,

$$
\|u \varsigma\|_{W^{s^{\prime}, r^{\prime}}(\Omega)} \leq c\|u\|_{W^{s^{\prime}, r^{\prime}(\Omega)}}\|\varsigma\|_{W^{s, r}(\Omega)}
$$

If $u \in W_{0}^{s^{\prime}, r^{\prime}}(\Omega)$, then $\varsigma u \in W_{0}^{s^{\prime}, r^{\prime}}(\Omega)$. Moreover, if in addition $s r \neq d+$ integer and $\varphi \in W^{s, r}(\Omega)$, then $\varsigma \nabla \varphi \in W^{s-1, r}(\Omega)$ and

$$
\|\varsigma \nabla \varphi\|_{W^{s-1, r}(\Omega)} \leq c\|\varphi\|_{W^{s, r}(\Omega)}\|\varsigma\|_{W^{s, r}(\Omega)} .
$$

Proof Since the embedding $W^{s, r}(\Omega) \hookrightarrow C(\Omega)$ is continuous, we have

$$
\begin{aligned}
& \int_{\Omega \times \Omega}|x-y|^{-d-r^{\prime} s^{\prime}}|\varsigma(x) u(x)-\varsigma(y) u(y)|^{r^{\prime}} d x d y \leq \\
& \int_{\Omega \times \Omega}|x-y|^{-d-r^{\prime} s^{\prime}}|u(x)|^{r^{\prime}}|\varsigma(x)-\varsigma(y)|^{r^{\prime}} d x d y+ \\
& \|\varsigma\|_{W^{s, r}(\Omega)}^{r^{\prime}} \int_{\Omega \times \Omega}|x-y|^{-d-r^{\prime} s^{\prime}}|u(x)-u(y)|^{r^{\prime}} d x d y \leq \\
& \int_{\Omega \times \Omega}|x-y|^{-d-r^{\prime} s^{\prime}}|u(x)|^{r^{\prime}}|\varsigma(x)-\varsigma(y)|^{r^{\prime}} d x d y+c\left(\|\varsigma\|_{W^{s, r}(\Omega)}\|u\|_{W^{s^{\prime}, r^{\prime}}(\Omega)}\right)^{r^{\prime}}
\end{aligned}
$$

Now set

$$
t^{-1}=\left(r^{\prime}\right)^{-1}-d^{-1} s^{\prime}, \quad \beta=d\left(s^{\prime}\right)^{-1}, \alpha^{-1}=1-r^{\prime} t^{-1}=r^{\prime} \beta^{-1}, \quad t, \beta, \alpha \in(1, \infty) .
$$


By virtue of (2.14) there is $\delta>0$ satisfying the inequalities

$$
s>1-s+\delta \equiv s^{\prime}+\delta \text { and } d-r s+r \delta \geq 0 .
$$

Fix such $\delta$ and set

$M(x)=\int_{\Omega}|x-y|^{-d-r^{\prime} s^{\prime}}|\varsigma(x)-\varsigma(y)|^{r^{\prime}} d y, N(x)=\int_{\Omega}|x-y|^{-d-\beta\left(s^{\prime}+\delta\right)}|\varsigma(x)-\varsigma(y)|^{\beta} d y$.

Since the embedding $W^{s^{\prime}, r^{\prime}}(\Omega) \hookrightarrow L^{t}(\Omega)$ is continuous for, we have from the Hölder inequality

$$
\begin{array}{r}
\int_{\Omega \times \Omega}|x-y|^{-d-r^{\prime} s^{\prime}}|u(x)|^{r^{\prime}}|\varsigma(x)-\varsigma(y)|^{r^{\prime}} d x d y= \\
\int_{\Omega}|u(x)|^{r^{\prime}} M(x) d x \leq\|u\|_{L^{t}(\Omega)}^{r^{\prime}}\|M\|_{L^{\alpha}(\Omega)} \leq c\|u\|_{W^{s^{\prime}, r^{\prime}(\Omega)}}^{r^{\prime}}\|M\|_{L^{\alpha}(\Omega)},
\end{array}
$$

Next note that

$$
|x-y|^{-d-r^{\prime} s^{\prime}}|\varsigma(x)-\varsigma(y)|^{r^{\prime}}=\left(|x-y|^{-d-\beta\left(s^{\prime}+\delta\right)}|\varsigma(x)-\varsigma(y)|^{\beta}\right)^{r^{\prime} / \beta}|x-y|^{-\gamma}
$$

where

$$
\gamma=d+s^{\prime} r^{\prime}-\frac{r^{\prime}}{\beta}\left[d+\left(s^{\prime}+\delta\right) \beta\right)=d \frac{\beta-r^{\prime}}{\beta}-r^{\prime} \delta \text { and } \gamma \frac{\beta}{\beta-r^{\prime}}<d .
$$

From this and Hölder inequality we conclude that

$$
M \leq N^{r^{\prime} / \beta} \int_{\Omega}|x-y|^{-\gamma \frac{\beta}{\beta-r^{\prime}}} d y \leq c(\delta, \Omega) N^{r^{\prime} / \beta},
$$

and $M^{\alpha} \leq N$. From this and (2.19) we obtain

$$
\begin{aligned}
& \int_{\Omega \times \Omega}|x-y|^{-d-r^{\prime} s^{\prime}}|u(x)|^{r^{\prime}}|\varsigma(x)-\varsigma(y)|^{r^{\prime}} d x d y \leq c\|u\|_{W^{s^{\prime}, r^{\prime}}(\Omega)}^{r^{\prime}}\left(\int_{\Omega} N d x\right)^{1 / \alpha} \\
& =c\|u\|_{W^{s^{\prime}, r^{\prime}(\Omega)}}^{r^{\prime}}\left(\int_{\Omega \times \Omega)}|x-y|^{-d-\beta\left(s^{\prime}+\delta\right)}|\varsigma(x)-\varsigma(y)|^{\beta} d y\right)^{r^{\prime} / \beta} \leq \\
& c\|u\|_{W^{s^{\prime}, r^{\prime}(\Omega)}}^{r^{\prime}}\|\varsigma\|_{W^{s^{\prime}+\delta, \beta}(\Omega)}^{r^{\prime}} .
\end{aligned}
$$

Notice that by virtue the identity $s^{\prime}=1-s$ and inequalities (2.18), we have $s>s^{\prime}+\delta$. Let us prove that the embedding $W^{s, r}(\Omega) \hookrightarrow W^{s^{\prime}+\delta, \beta}(\Omega)$ is bounded. If $r\left(s-s^{\prime}-\delta\right) \geq d$ it is bounded for all $\beta<\infty$. For $r\left(s-s^{\prime}-\delta\right) \leq d$ it is bounded if and only if $\beta$ satisfies the inequality

$$
\frac{1}{\beta} \equiv \frac{s^{\prime}}{d} \geq \frac{1}{r}-\frac{2 s-1-\delta}{d}
$$

which obviously follows from (2.18). Thus we get $\|\varsigma\|_{W^{1-s+\delta, \beta}(\Omega)} \leq\|\varsigma\|_{W^{s, r}(\Omega)}$. Combining this result with (2.17) and (2.20) we finally obtain

$$
\int_{\Omega \times \Omega}|x-y|^{-d-r^{\prime} s^{\prime}}|\varsigma(x) u(x)-\varsigma(y) u(y)|^{r^{\prime}} d x d y \leq c\|u\|_{W^{s^{\prime}, r^{\prime}(\Omega)}}^{r^{\prime}}\|\varsigma\|_{W^{s, r}(\Omega)}^{r^{\prime}},
$$


which yields (2.15).

Now assume that $u \in W_{0}^{s^{\prime}, r^{\prime}}(\Omega)$. Recall, see [25], that a function $u \in W_{0}^{s^{\prime}, r^{\prime}}(\Omega)$ belongs to $W_{0}^{s^{\prime}, r^{\prime}}(\Omega)$ if and only if $u \in W^{s^{\prime}, r^{\prime}}(\Omega)$ and $\operatorname{dist}(x, \partial \Omega)^{-s^{\prime}} \in L^{r^{\prime}}(\Omega)$. Since the function $\varsigma$ is bounded, the function $u \varsigma$ belongs to $W_{0}^{s^{\prime}, r^{\prime}}(\Omega)$ for $u \in$ $W_{0}^{s^{\prime}, r^{\prime}}(\Omega)$. Moreover, since by virtue of $(2.14) s^{\prime} \neq r^{\prime}+$ integer , we have

$$
\|u \varsigma\|_{W_{0}^{s^{\prime}, r^{\prime}}(\Omega)} \leq c\|u\|_{W_{0}^{s^{\prime}, r^{\prime}(\Omega)}}\|\varsigma\|_{W^{s, r}(\Omega)} .
$$

Finally notice that condition $(2.14)$ yields the relation $-s^{\prime} \neq 1 / r+$ integer. Hence the identity $\langle u, \varsigma \nabla \varphi\rangle=\langle u \varsigma, \nabla \varphi\rangle$ determines a continuous linear functional on $W^{-s^{\prime}, r}(\Omega)$ and the lemma follows.

Transport equation. The following lemma is a particular case of the general results on solvability of transport equations in fractional Sobolev spaces, see [20] and [21]. It concerns with the existence and uniqueness of solutions to the following boundary value problems for the first order differential equations

$$
\begin{aligned}
\mathscr{L} \varphi & :=\mathbf{u} \nabla \varphi+\sigma \varphi=f \text { in } \Omega, \quad \varphi=0 \quad \text { on } \Sigma_{\text {in }}, \\
\mathscr{L}^{*} \varphi^{*} & :=-\operatorname{div}\left(\varphi^{*} \mathbf{u}\right)+\sigma \varphi^{*}=f \quad \text { in } \Omega, \quad \varphi^{*}=0 \text { on } \Sigma_{\text {out }} .
\end{aligned}
$$

Lemma 2.3. Let a vector field $\mathbf{u} \in C^{1}(\Omega)$ satisfies the boundary condition

$$
\mathbf{u}=\mathbf{U} \text { on } \Sigma, \quad \mathbf{u}=0 \text { on } \mathcal{S},
$$

where $\mathbf{U}$ is a given constant vector field. Furthermore, assume that the exponents $s, r$ satisfy the inequalities. Then there are positive constants $\sigma^{*}$ and $\delta^{*}$, depending only on $\Sigma, \mathbf{U}, s, r$ and $\|\mathbf{u}\|_{C^{1}(\Omega)}$, with the following property. If $\|\operatorname{div} \mathbf{u}\|_{W^{s, r}(\Omega)}+$ $\|\operatorname{div} \mathbf{u}\|_{L^{\infty}(\Omega)} \leq \delta^{*}, \sigma>\sigma^{*}$, then for any $f \in W^{s, r}(\Omega)$ each of problems (2.21) and (2.22) has a unique solution $\varphi, \varphi^{*} \in W^{s, r}(\Omega)$, which admits the estimates

$$
\|\varphi\|_{W^{s, r}(\Omega} \leq C\|f\|_{W^{s, r}(\Omega)}, \quad\left\|\varphi^{*}\right\|_{W^{s, r}(\Omega)} \leq C\|f\|_{W^{s, r}(\Omega)} .
$$

The constant $C$ depends only on $\|\mathbf{u}\|_{C^{1}(\Omega)}, r, s \sigma, \mathbf{U}$ and $\Omega$.

Solvability of adjoint state equations. Let us consider the boundary value problem

$$
\begin{gathered}
\Delta \mathbf{h}-\nabla g-R \varrho \nabla \mathbf{u h}+R \varrho \mathbf{u} \nabla \mathbf{h}+\varsigma \nabla \varphi+\zeta \nabla v=\mathbf{F}, \\
\operatorname{div} \mathbf{h}-\Pi\left(b_{12} \varsigma+b_{22} g+\kappa b_{32} l\right)=G, \\
-\operatorname{div}(\mathbf{u} \varsigma)+\sigma \varsigma-R(\mathbf{u} \nabla \mathbf{u}) \cdot \mathbf{h}-b_{21} g-b_{11} \varsigma-\varkappa b_{31} l=P, \\
\mathbf{u} \nabla v+\sigma v-\varkappa b_{34} l=Q, \\
l-\int_{\Omega} b_{13} \varsigma d x=n
\end{gathered}
$$

$$
\mathbf{h}=0 \text { on } \partial B, \quad \mathbf{h}=\mathbf{a} \text { on } \mathcal{S}, \quad \varsigma=0 \text { on } \Sigma_{\text {out }}, \quad v=0 \text { on } \Sigma_{\text {in }}, \quad g=\Pi g .
$$

The following proposition is the main result of this section

Proposition 2.4. Assume that domain $\Omega$ satisfies Condition 1.1, and the exponents $s, r$ satisfy conditions (2.3) and inequalities $s \neq 1 / r+$ integer. Furthermore, assume that a solution $(\mathbf{v}, \varphi, \varsigma)$ to problem (1.13) with $\varepsilon=0$ meets all requirements of Proposition 1.2. Then there exist $\mu>0, c_{0}$ depending only on $\mathbf{U}, \Omega$ and $s, r$, such that for all

$$
R+\lambda^{-1} \leq \mu
$$


and $\mathbf{F} \in H^{s-1, r}(\Omega), G \in \Pi W^{s, r}(\Omega), P, Q \in W^{s, r}(\Omega), n \in \mathbb{R}, \mathbf{a} \in \mathbb{R}^{d}$ problem (2.25) has the only solution which admits the estimate

$$
\begin{aligned}
& \|\mathbf{h}\|_{W^{1+s, r}(\Omega)}+\|g\|_{W^{s, r}(\Omega)}+\|s\|_{W^{s, r}(\Omega}+\|v\|_{W^{s, r}(\Omega}+|l| \leq \\
& c_{0}\left(\|\mathbf{F}\|_{W^{s-1, r}(\Omega)}+\|G\|_{W^{s, r}(\Omega)}+\|P\|_{W^{s, r}(\Omega}+\|Q\|_{W^{s, r}(\Omega}+|n|+|\mathbf{a}|\right) .
\end{aligned}
$$

Proof. It suffices to prove the proposition for $\mathbf{a}=0$. We begin with the observation that by virtue of inequalities $(2.3)$, the spaces $W^{s, r}(\Omega)$ and $W^{1+s, r}(\Omega)$ are the Banach algebras and the embeddings $X^{s, r} \hookrightarrow W^{s, r}(\Omega), Y^{s, r} \hookrightarrow W^{s, r}(\Omega)$ are continuous. It follows from this, inequalities (1.16), (2.27) and formulae (1.19) that coefficients $b_{i j}$ admit the estimates

$$
\left\|b_{13}\right\|_{W^{s, r}(\Omega)}+\left\|b_{21}\right\|_{W^{s, r}(\Omega)}+\left\|b_{23}\right\|_{W^{s, r}(\Omega)} \leq c(s, r, \sigma, \Omega)
$$

and

$$
\begin{aligned}
& \left\|b_{11}\right\|_{W^{s, r}(\Omega)}+\left\|b_{12}\right\|_{W^{s, r}(\Omega)}+\left\|b_{10}\right\|_{W^{s, r}(\Omega)}+ \\
& \left\|b_{22}\right\|_{W^{s, r}(\Omega)}+\left\|b_{20}\right\|_{W^{s, r}(\Omega)}+\left\|b_{3 i}\right\|_{W^{s, r}(\Omega)} \leq c(s, r, \sigma, \Omega) \mu
\end{aligned}
$$

Let us consider the truncated problem

$$
\begin{gathered}
\Delta \mathbf{h}-\nabla g+\zeta \nabla v=\mathbf{F}, \quad \operatorname{div} \mathbf{h}=G, \\
-\operatorname{div}(\mathbf{u} \varsigma)+\sigma \varsigma-b_{21} g=P, \\
\mathbf{u} \nabla v+\sigma v=Q, \\
l-\int_{\Omega} b_{13} \varsigma d x=n \\
\mathbf{h}=0 \text { on } \partial \Omega, \quad \varsigma=0 \text { on } \Sigma_{\text {out }}, \quad v=0 \text { on } \Sigma_{\text {in }}, \quad g=\Pi g .
\end{gathered}
$$

This system is of triangle nature. Using Lemma 2.3 we conclude that in the assumptions of Proposition 1.2 we can choose $\sigma^{*}$, depending only on $\Omega, s, r$ and $\mathbf{U}$, so that for every fixed $\sigma \geq \sigma^{*}$ equation (2.30c) has the only solution satisfying boundary condition (2.30e) and the inequality

$$
\|v\|_{W^{s, r}(\Omega)} \leq c\|Q\|_{W^{s, r}(\Omega)}
$$

Next, using estimate (1.16) and Lemma 2.2 we conclude that

$$
\|\zeta \nabla v\|_{W^{s-1, r}(\Omega)} \leq c\|v\|_{W^{s, r}(\Omega)} \leq c\|Q\|_{W^{s, r}(\Omega)} .
$$

Combining this result with Lemma 2.1 we obtain that equations (2.30a) have a unique solution $(\mathbf{h}, g) \in W^{s+1, r}(\Omega) \times \Pi W^{s, r}(\Omega)$ satisfying boundary condition (2.30e) and the inequality

$$
\|\mathbf{h}\|_{W^{s+1, r}(\Omega)}+\|g\|_{W^{s, r}(\Omega)} \leq c\left(\|Q\|_{W^{s, r}(\Omega)}+\|G\|_{W^{s, r}(\Omega)}+\|\mathbf{F}\|_{W^{s-1, r}(\Omega)}\right) .
$$

Applying Lemma 2.3 and using estimates (2.28) we obtain that equations (2.30b) and $(2.30 \mathrm{~d})$ have solutions satisfying the inequalities

$$
\|\varsigma\|_{W^{s, r}(\Omega)}+|l| \leq c\left(\|P\|_{W^{s, r}(\Omega)}+\|Q\|_{W^{s, r}(\Omega)}+\|G\|_{W^{s, r}(\Omega)}+\|\mathbf{F}\|_{W^{s-1, r}(\Omega)}+|n|\right) .
$$

It follows from this that the mapping $(\mathbf{F}, G, P, Q, n) \rightarrow(\mathbf{h}, g, \varsigma, v, l)$ determined by equations (2.30) determines the continuous operator $\mathfrak{A}:\left(W^{s-1, r}(\Omega) \times \Pi W^{s, r}(\Omega) \times\right.$ 
$W^{s, r}(\Omega)^{2} \times \mathbb{R} \mapsto W^{s+1, r}(\Omega) \times \Pi W^{s, r}(\Omega) \times W^{s, r}(\Omega)^{2} \times \mathbb{R}$. Let us consider the operator $\mathfrak{B}=\left(\mathfrak{B}_{1}, \mathfrak{B}_{2}, \mathfrak{B}_{3}, \mathfrak{B}_{4}\right)$ defined by the equalities

$$
\begin{array}{r}
\mathfrak{B}_{1}(\mathbf{h}, g, \varsigma, v, l)=-R \varrho \nabla \mathbf{u h}+R \varrho \mathbf{u} \nabla \mathbf{h}+\varsigma \nabla \varphi, \\
\mathfrak{B}_{2}(\mathbf{h}, g, \varsigma, v, l)=-\Pi\left(b_{12} \varsigma+b_{22} g+\kappa b_{32} l\right), \\
\mathfrak{B}_{3}(\mathbf{h}, g, \varsigma, v, l)=-R(\mathbf{u} \nabla \mathbf{u}) \cdot \mathbf{h}-b_{11} \varsigma-\varkappa b_{31} l, \quad \mathfrak{B}_{4}(\mathbf{h}, g, \varsigma, v, l)=0
\end{array}
$$

It follows from estimate (1.16) and Lemma 2.2 that

$$
\|\varsigma \nabla \varphi\|_{W^{s-1, r}(\Omega)} \leq c\|\varsigma\|_{W^{s, r}(\Omega)}\|\varphi\|_{W^{s, r}(\Omega)} \leq c \mu\|\varsigma\|_{W^{s, r}(\Omega)} .
$$

Combining this result with estimates (1.16), (2.29) we obtain that the norm of the operator $\mathfrak{B}:\left(W^{s-1, r}(\Omega) \times \Pi W^{s, r}(\Omega) \times W^{s, r}(\Omega)^{2} \times \mathbb{R} \mapsto W^{s+1, r}(\Omega) \times \Pi W^{s, r}(\Omega) \times\right.$ $W^{s, r}(\Omega)^{2} \times \mathbb{R}$ does not exceed $c \mu$. Since equations (2.25) are equivalent to the operator equation

$$
\left(\mathbf{I}+\mathfrak{A}^{-1} \mathfrak{B}\right)(\mathbf{h}, g, \varsigma, v, l)=\mathfrak{A}^{-1}(\mathbf{F}, G, P, Q, n),
$$

the assertion of the lemma results from the contraction mapping principle.

\section{Normal COORDinates}

For any point $\omega \in \mathcal{S}$ denote by $\mathbf{n}$ the unique outward normal vector to $\mathcal{S}$ at point $\omega$. Since $\mathcal{S}$ is a smooth manifold, there exist a neighborhood $\mathcal{O}$ of $\mathcal{S}$ and a number $a>0$ so that the mapping $\left(\omega, t_{3}\right) \rightarrow \omega+t_{3} \mathbf{n}(\omega)$ takes diffeomorphically the set $\mathcal{S} \times[-a, a] \rightarrow \mathcal{O}$.

Let us calculate the derivatives and Jacobian of this mapping. To this end we fix an arbitrary point $\omega_{0} \in \mathcal{S}$. In some neighborhood $\mathcal{O}_{0}$ of this point, the surface $\mathcal{S}$ admits the parametric representation

$$
\mathcal{S} \cap \mathcal{O}_{0}: \omega=\mathbf{r}\left(t_{1}, t_{2}\right), \quad\left|t_{i}\right| \leq a, \quad \mathbf{r}(0,0)=\omega_{0},
$$

where the vector-valued function $\mathbf{r}\left(t_{1}, t_{2}\right)$ belong to the class $C^{\infty}$ in the rectangular

$$
Q=\left\{\left(t_{1}, t_{2}\right):\left|t_{i}\right| \leq a, i=1,2\right\},
$$

for an appropriate choice of $a$ independent of $\omega_{0}$. We will write

$$
\mathbf{t}=\left(t_{1}, t_{2}, t_{3}\right), \quad \bar{t}=\left(t_{1}, t_{2}\right) \text { so that } \mathbf{t}=\left(\bar{t}, t_{3}\right) .
$$

and introduce the moving frame

$$
\mathbf{e}_{i}(\bar{t})=\partial_{t_{i}} \mathbf{r}(\bar{t}), \quad i=1,2, \quad \mathbf{e}_{3}(\bar{t})=\mathbf{n}(\mathbf{r}(\bar{t}))=\frac{1}{\left|\mathbf{e}_{1} \times \mathbf{e}_{2}\right|} \mathbf{e}_{1} \times \mathbf{e}_{2} .
$$

Thus the mapping

$$
X: \mathbf{t} \mapsto \mathbf{r}(\bar{t})+t_{3} \mathbf{e}_{3}(\bar{t}) \equiv \omega+t_{3} \mathbf{n}(\omega)
$$

takes diffeomorphically

$$
Q \times[-a, a] \mapsto \mathcal{O}_{0} \text { and } Q \times[-a, 0] \mapsto \mathcal{O}_{0} \cap \Omega
$$

Recall the denotations

$$
g_{i j}=\mathbf{e}_{i}(\bar{t}) \cdot \mathbf{e}_{j}(\bar{t}), \quad b_{i j}=\mathbf{e}_{3}(\bar{t}) \cdot \partial_{t_{i} t_{j}}^{2} \mathbf{r}(\bar{t})
$$

for the coefficients of the first and second fundamental form of the surface $\mathcal{S}$ and set

$$
g=\operatorname{det}\left(g_{i j}\right), \quad\left(g^{\alpha \beta}\right)=\left(g_{\alpha \beta}\right)^{-1} .
$$


In this notation, the mean and Gauss curvatures of $\mathcal{S}$ are determined by the equalities

$$
H=\frac{1}{2}\left(b_{1}^{1}+b_{2}^{2}\right), \quad K=b_{1}^{1} b_{2}^{2}-b_{2}^{1} b_{1}^{2}, \text { where } b_{j}^{i}=g^{i \alpha} b_{\alpha j}
$$

Using the Weigharten equations

$$
\partial_{t_{j}} \mathbf{e}_{3}=-b_{j}^{i} \mathbf{e}_{i} \text { with } i, j=1,2,
$$

we obtain the expression for the columns of the Jacobi matrix $X^{\prime}(\mathbf{t})$

$$
\partial_{t_{j}} X=\mathbf{e}_{j}-t_{3}\left(b_{j}^{1} \mathbf{e}_{1}+b_{j}^{2} \mathbf{e}_{2}\right), j=1,2, \quad \partial_{t_{3}} X=\mathbf{e}_{3} .
$$

In order to derive the expression to the inverse of $X^{\prime}(\mathbf{t})$ we use the identity

$$
\begin{aligned}
& \left(X^{\prime-1}\right)^{*} \equiv\left[\nabla_{x} t_{1}(X(\mathbf{t})), \nabla_{x} t_{2}(X(\mathbf{t})), \nabla_{x} t_{3}(X(\mathbf{t}))\right]= \\
& \left(\operatorname{det} X^{\prime}\right)^{-1}\left[\partial_{t_{2}} X \times \partial_{t_{3}} X, \partial_{t_{3}} X \times \partial_{t_{1}} X, \partial_{t_{1}} X \times \partial_{t_{2}} X,\right] .
\end{aligned}
$$

Next note that for $j=1,2$,

$\left.\mathbf{e}_{j} \times \mathbf{e}_{3}=g^{-1 / 2} \mathbf{e}_{j} \times\left(\mathbf{e}_{1} \times \mathbf{e}_{2}\right)=g^{-1 / 2}\left(\left(\mathbf{e}_{j} \cdot \mathbf{e}_{2}\right) \mathbf{e}_{1}-\left(\mathbf{e}_{j} \cdot \mathbf{e}_{1}\right) \mathbf{e}_{2}\right)\right)=g^{-1 / 2}\left(g_{j 2} \mathbf{e}_{1}-g_{j 1} \mathbf{e}_{2}\right)$

and $\mathbf{e}_{1} \times \mathbf{e}_{2}=g^{1 / 2} \mathbf{e}_{3}$. From this and (3.2), (3.3) we obtain

$$
\partial_{t_{j}} X \times \partial_{t_{3}} X=g^{-1 / 2}\left(g_{j 2} \mathbf{e}_{1}-g_{j 1} \mathbf{e}_{2}\right)-t_{3} g^{-1 / 2}\left(b_{j 2} \mathbf{e}_{1}-b_{j 1} \mathbf{e}_{2}\right) .
$$

and

$$
\partial_{t_{1}} X \times \partial_{t_{2}} X=g^{1 / 2}\left(1+2 H t_{3}+K t_{3}^{2}\right)
$$

In particular we have the following expression for the Jacobian

$$
\operatorname{det} X^{\prime} \equiv\left(\partial_{t_{1}} X \times \partial_{t_{2}} X\right) \cdot \partial_{t_{3}} X=g^{1 / 2}\left(1+2 H t_{3}+K t_{3}^{2}\right) .
$$

We also get the following expression to the lines of the matrix $\left(X^{\prime}\right)^{-1}$ ( the columns of the matrix $\left.\left(X^{\prime-1}\right)^{*}\right)$

$$
\begin{aligned}
& \left(\nabla_{x} t_{1}\right)(X(\mathbf{t}))=g^{-1}\left(1+2 H t_{3}+K t_{3}^{2}\right)^{-1}\left\{g_{22} \mathbf{e}_{1}-g_{21} \mathbf{e}_{2}-t_{3}\left(b_{22} \mathbf{e}_{1}-b_{21} \mathbf{e}_{2}\right)\right\}, \\
& \left(\nabla_{x} t_{2}\right)(X(\mathbf{t}))=-g^{-1}\left(1+2 H t_{3}+K t_{3}^{2}\right)^{-1}\left\{\left(g_{12} \mathbf{e}_{1}-g_{11} \mathbf{e}_{2}\right)-t_{3}\left(b_{12} \mathbf{e}_{1}-b_{11} \mathbf{e}_{2}\right)\right\}, \\
& \left(\nabla_{x} t_{3}\right)(X(\mathbf{t}))=\mathbf{e}_{3} .
\end{aligned}
$$

Conformal coordinates The formulae can be essentially simplified if the parametrization $x=\mathbf{r}(\bar{t})$ is conformal. This means that

$$
g_{12}=0, \quad g_{11}=g_{22}=g^{1 / 2}
$$

In this case the expression for the lines of the inverse Jacobi becomes

$$
\begin{aligned}
& \left(\nabla_{x} t_{1}\right)(X(\mathbf{t}))=g^{-1}\left(1+2 H t_{3}+K t_{3}^{2}\right)^{-1}\left\{g^{1 / 2} \mathbf{e}_{1}-t_{3}\left(b_{22} \mathbf{e}_{1}-b_{21} \mathbf{e}_{2}\right)\right\}, \\
& \left(\nabla_{x} t_{2}\right)(X(\mathbf{t}))=g^{-1}\left(1+2 H t_{3}+K t_{3}^{2}\right)^{-1}\left\{g^{1 / 2} \mathbf{e}_{2}-t_{3}\left(b_{11} \mathbf{e}_{2}-b_{12} \mathbf{e}_{1}\right)\right\}, \\
& \left(\nabla_{x} t_{3}\right)(X(\mathbf{t}))=\mathbf{e}_{3} .
\end{aligned}
$$

In particular, we have the following expression for the Jacobi matrix and its inverse at the sheet $\mathcal{S} \cap \mathcal{O}_{0}$

$$
X^{\prime}(\mathbf{t})=\left[\mathbf{e}_{1}, \mathbf{e}_{2}, \mathbf{e}_{3}\right], \quad\left(X^{\prime-1}\right)^{*}(\mathbf{t})=\left[g^{-1 / 2} \mathbf{e}_{1}, g^{-1 / 2} \mathbf{e}_{2}, \mathbf{e}_{3}\right] .
$$


3.1. Surface perturbation. Choose an arbitrary function $f \in C^{\infty}(\mathcal{S})$. Without loss of generality we can assume that $f$ is extended over $\mathbb{R}^{3}$ and the extension belongs to the class $C^{\infty}\left(\mathbb{R}^{3}\right)$ Let us consider the immersion

$$
\mathcal{S}_{\tau}: x=\omega+\tau f(\omega) \mathbf{n}(\omega), \omega \in \mathcal{S},
$$

where $\tau \in[0, a]$ is a small parameter. For all small $\tau$ this immersion is the embedding and we obtain the family of smooth surfaces $\mathcal{S}_{\tau}$ which can be regarded as a normal perturbation of $\mathcal{S}$ generated by the normal shift $f \mathbf{n}$. Now our task is to extend the mapping (3.8) over $\mathbb{R}^{3}$. To this end fix a function $\chi \in C_{0}^{\infty}(\mathbb{R})$ satisfying the relations

$$
\chi(s)=1 \text { for }|s| \leq 1 / 2, \quad \chi(t)=0 \text { for }|s| \geq 1 .
$$

Introduce a vector field $\mathbf{T}_{\tau}(x)$ defined in the neighborhood $\mathcal{O}$ of the surface $\partial S$ by the relations

$\mathbf{T}_{\tau}(x)=\mathbf{T}_{\tau}\left(\omega+t_{3} \mathbf{n}(\omega)\right)=f(\omega) \chi\left(t_{3} / \tau\right) \mathbf{n}(\omega)$, where $t_{3}=t_{3}(x) \equiv \operatorname{dist}(x, \mathcal{S})$.

Since the vector field $\mathbf{T}_{\tau}$ vanishes for $t_{3} \geq a$, it, being extended by 0 over $\mathbb{R}^{3}$, belongs to the class $C^{\infty}\left(\mathbb{R}^{3}\right)$. Hence for all small $\varepsilon$, the mapping $x \rightarrow x+\varepsilon \mathbf{T}_{\tau}(x)$ determines the diffeomoprhism of $\mathbb{R}^{3}$. Moreover this diffeomorphism is equal to the identical mapping outside of $\tau$ - neighborhood of $\mathcal{S}$.

Now we consider in more details the structure of the derivative of $T_{\tau}^{\prime}$ and the related matrix $\mathbf{D}_{\tau}$. In order to formulate the corresponding result it is convenient introduce the notation $\mathbf{M}(\mathbf{t})$ for the Jacobi matrix $X^{\prime}()$.

Lemma 3.1. Under the above assumptions,

$$
\begin{aligned}
& \mathbf{T}_{\tau}^{\prime}(x)=\frac{1}{\tau} \chi^{\prime}\left(t_{3} / \tau\right) f(\omega) \mathbf{n}(\omega) \otimes \mathbf{n}(\omega)+ \\
& \chi\left(t_{3} / \tau\right) \mathbf{n}(\omega) \otimes \nabla_{t a n} f(\omega)+\chi\left(t_{3} / \tau\right) \mathbb{S},
\end{aligned}
$$

where $t_{3}=t_{3}(x)$,

$$
\nabla_{\text {tan }} f(\omega)=\left.(\mathbf{I}-\mathbf{n}(\omega) \otimes \mathbf{n}(\omega)) \nabla f(x)\right|_{t_{3}=0}
$$

is the orthogonal projection of the gradient $f$ onto the tangential plane to $\mathcal{S}$, and elements $\mathbf{S}_{i j}$ of the matrix $\mathbf{S}$ in the local coordinates $\mathbf{t}$ are defined by the equalities

$$
\mathbf{S}_{i j}=\chi\left(t_{3} / \tau\right) f(\omega) \sum_{\alpha=1,2} W_{j}^{\alpha}(\omega) e_{i \alpha}, \quad W_{j}^{\alpha}(\bar{t})=-b_{\beta}^{\alpha}\left(\mathbf{M}^{-1}\right)_{\beta j},
$$

in which $e_{i \alpha}$ is the ith component of the vector $\mathbf{e}_{\alpha}$. Moreover, we have

$$
\operatorname{Tr} \mathbf{T}_{\tau}^{\prime}=\frac{1}{\tau} \chi^{\prime}\left(t_{3} / \tau\right) f(\omega)-2 \chi f\left(t_{3} / \tau\right) H(\omega),
$$

where $H(\omega)$ is the mean curvature of $\mathcal{S}$. The matrix $\mathbf{D}$ admits the representation

$$
\begin{array}{r}
\mathbf{D}_{\tau}=\left(\frac{1}{\tau} \chi^{\prime}\left(t_{3} / \tau\right) f(\omega)-2 \chi f\left(t_{3} / \tau\right) H(\omega)\right) \mathbf{I}- \\
\frac{1}{\tau} \chi^{\prime}\left(t_{3} / \tau\right) f(\omega) \mathbf{n}(\omega) \otimes \mathbf{n}(\omega)+\chi\left(t_{3} / \tau\right) \mathbf{n}(\omega) \otimes \nabla_{t a n} f(\omega)+\chi\left(t_{3} / \tau\right) \mathbf{S} .
\end{array}
$$

The matrixes $\mathbf{T}_{\tau}^{\prime}$ and $\mathbf{D}_{\tau}$ satisfy the boundary conditions

$$
\mathbf{T}_{\tau}^{\prime}=\mathbf{n}(\omega) \otimes \nabla_{\text {tan }} f(\omega)+\mathbf{S}, \quad \mathbf{D}_{\tau}=-2 f H \mathbf{I}-\mathbf{n}(\omega) \otimes \nabla_{\text {tan }} f(\omega)-\mathbf{S} \text { on } \mathcal{S} .
$$


Proof. By virtue of the partition of unit approach, it suffices to prove the lemma in the neighborhood of $\mathcal{O}_{0}$ of an arbitrary point. $\omega_{0} \in \mathcal{S}$. Let $\mathbf{t} \in Q \times[-a, a]$ be a local coordinate system in this neighborhood, such that $\bar{t}$ is a local conformal coordinates on the manofold $\mathcal{S} \cap \mathcal{O}_{0}$. In this coordinates we have

$$
\partial_{x_{i}} \mathbf{T}_{\tau}=\frac{1}{\tau} \chi^{\prime} f \partial_{x_{i}} t_{3} \mathbf{n}+\chi \partial_{x_{p}} f \partial_{t_{q}}(\mathbf{r})_{p} \partial_{x_{i}} t_{q} \mathbf{n}+\mathbf{n}+\chi f \partial_{x_{i}} t_{p} \partial_{t_{p}} \mathbf{n}
$$

where $(\mathbf{r})_{p}$ is the $p$ th component of the vector-valued function $\mathbf{r}(\bar{t})$. Noting that $\nabla_{x} t_{3}=\mathbf{e}_{3}(\mathbf{t})=\mathbf{n}(x(\mathbf{t}))$ we arrive at the first basic identity

$$
\frac{1}{\tau} \chi^{\prime} f \partial_{x_{i}} t_{3} \mathbf{n}=\frac{1}{\tau} \chi^{\prime} f n_{i} \mathbf{n} .
$$

Next we have

$$
\begin{array}{r}
\partial_{x_{p}} f \partial_{t_{q}}(\mathbf{r})_{p} \partial_{x_{i}} t_{q} \equiv \sum_{q=1,2} \partial_{x_{p}} f \partial_{t_{q}}(\mathbf{r})_{p} \partial_{x_{i}} t_{q} \\
\equiv \sum_{q=1,2} \partial_{x_{p}} f M_{p q} N q_{i}=\partial_{x_{i}} f-M_{p 3} N_{3 i} \partial_{x_{p}} f=\partial_{x_{i}} f-n_{p} n_{i} \partial_{x_{p}} f
\end{array}
$$

which leads to the second identity

$$
\chi \partial_{x_{p}} f \partial_{t_{q}}(\mathbf{r})_{p} \partial_{x_{i}} t_{q} \mathbf{n}=\left(\nabla_{t a n} f\right)_{i} \mathbf{n}
$$

where $\left(\nabla_{\text {tan }} f\right)_{i}$ is the $i$ th component of the vector $\nabla_{\text {tan }} f$. Using the Weigharten equation we also get the third identity

$$
\chi f \partial_{x_{i}} t_{p} \partial_{t_{p}} \mathbf{n}=-\chi f \sum_{i=1,2} b_{\beta}^{\alpha}\left(\mathbf{M}^{-1}\right)_{\beta i} \mathbf{e}_{\alpha}=: \chi f \sum_{i=1,2} W_{i}^{\alpha} \mathbf{e}_{\alpha} .
$$

Combining (3.1)-(3.17) we finally obtain (3.10). Since $e_{i, \alpha}=M_{i \alpha}$, we have

$$
\operatorname{Tr} \mathbf{S}=\chi f \sum_{i=1,2} \sum_{\alpha=1,2} W_{i}^{\alpha} e_{i \alpha}=-\chi f \sum_{\alpha=1,2} b_{\beta}^{\alpha}\left(\mathbf{M}^{-1}\right)_{\beta i} \mathbf{M}_{i \alpha}=-\chi f b_{\alpha}^{\alpha}=-2 \chi f H
$$

which along with (3.10) and the identity $\nabla_{\text {tan }} f \cdot \mathbf{n}=0$ leads to the representation (3.11). Formula (3.12) is an obvious consequence of (3.10) and (3.11). It remains to note that since $\chi^{\prime}=0$ and $\chi=1$ on $\mathcal{S}$ boundary conditions (3.13) follows from (3.11) and (3.12).

\section{Limiting RELATiOnS}

In this section we establish some auxiliary facts on the singular limits of integrals, with the integrands depending on the derivatives of $T_{\tau}$

Lemma 4.1. For any $\psi \in C(\Omega)$,

$$
\begin{gathered}
\lim _{\tau \searrow 0} \int_{\Omega} \psi(x) \mathbf{T}_{\tau}^{\prime}(x) d x=\int_{\mathcal{S}} \psi(\omega) f(\omega) \mathbf{n} \otimes \mathbf{n} d \omega, \\
\quad \lim _{\tau \searrow 0} \int_{\Omega} \psi(x) \operatorname{Tr} \mathbf{T}_{\tau}^{\prime}(x) d x=\int_{\mathcal{S}} \psi(\omega) f(\omega) d s
\end{gathered}
$$


Proof. Using the partition of unit we can assume that spt $\psi$ concentrated in the neighborhood of point $\omega_{0} \in \mathcal{S}$ and hence

$$
\int_{\Omega} \psi(x) \mathbf{T}_{\tau}^{\prime}(x) d x=\int_{Q} \int_{-\tau}^{0} \tilde{\psi}(\mathbf{t}) T_{\tau}^{\prime}(X(\mathbf{t})) \operatorname{det} X^{\prime}(\mathbf{t}) d \mathbf{t}
$$

where $\tilde{\psi}(\mathbf{t})=\psi(X(\mathbf{t}))$ and $Q=[-a, a]^{2}$. Using the identity (3.10) and formula (3.4) we obtain

$$
\mathbf{T}_{\tau}^{\prime}(X(\mathbf{t})) \operatorname{det} X^{\prime}(\mathbf{t})=\frac{1}{\tau} \chi^{\prime}\left(t_{3} / \tau\right) f(\omega(\bar{t})) \mathbf{e}_{3}(\bar{t}) \otimes \mathbf{e}_{3}(\bar{t}) g^{1 / 2}(\bar{t})+\mathbf{R}(\mathbf{t}),
$$

where

$$
\begin{array}{r}
\mathbf{R}(\mathbf{t})=\frac{t_{3}}{\tau} \chi^{\prime}\left(t_{3} / \tau\right) f(\omega(\bar{t})) \mathbf{e}_{3}(\bar{t}) \otimes \mathbf{e}_{3}(\bar{t})\left(2 H+t_{3} K\right)+ \\
\left(\chi\left(t_{3} / \tau\right) \mathbf{e}_{3}(\mathbf{t}) \otimes \nabla_{t a n} f(\omega(\bar{t}))+\chi\left(t_{3} / \tau\right) \mathbf{S}(\mathbf{t})\right) g^{1 / 2}\left(1+t_{3} H+t_{3}^{2} K\right)
\end{array}
$$

Since the functions $f, \nabla f$ and elements of the matrix $\mathbf{S}$ are uniformly bounded in $Q \times[-\tau, 0]$ we have

$$
\int_{Q} \int_{-\tau}^{0} \tilde{\mid} \psi(\mathbf{t}) \mathbf{R}(\mathbf{t}) \mid d \mathbf{t} \leq c \tau \rightarrow 0 \text { as } \tau \rightarrow 0
$$

Thus we get

$$
\lim _{\tau \searrow 0} \int_{\Omega} \psi(x) \mathbf{T}_{\tau}^{\prime}(x) d x=\lim _{\tau \searrow 0} \frac{1}{\tau} \int_{Q} \int_{-\tau}^{0} \tilde{\psi}(\mathbf{t}) \chi^{\prime}\left(t_{3} / \tau\right) f(\omega(\bar{t})) \mathbf{e}_{3}(\bar{t}) \otimes \mathbf{e}_{3}(\bar{t}) g^{1 / 2}(\bar{t}) d \mathbf{t}
$$

Noting that

$$
\lim _{\tau \searrow 0} \frac{1}{\tau} \int_{Q} \int_{-\tau}^{0}[\tilde{\psi}(\mathbf{t})-\tilde{\psi}(\bar{t}, 0)] \chi^{\prime}\left(t_{3} / \tau\right) f(\omega(\bar{t})) \mathbf{e}_{3}(\bar{t}) \otimes \mathbf{e}_{3}(\bar{t}) g^{1 / 2}(\bar{t}) d \mathbf{t}=0
$$

and using the relations $\mathbf{e}_{3}(\bar{t})=\mathbf{n}(\omega(\bar{t}))$ and $g^{1 / 2} d t_{1} d t_{2}=d s$ we obtain

$$
\begin{aligned}
& \lim _{\tau \searrow 0} \int_{\Omega} \psi(x) \mathbf{T}_{\tau}^{\prime}(x) d x=\lim _{\tau \searrow 0} \frac{1}{\tau} \int_{Q} \int_{-\tau}^{0} \tilde{\psi}(\bar{t}, 0) \chi^{\prime}\left(t_{3} / \tau\right) f(\omega(\bar{t})) \mathbf{e}_{3}(\bar{t}) \otimes \mathbf{e}_{3}(\bar{t}) g^{1 / 2}(\bar{t}) d \mathbf{t} \\
& =\left(\lim _{\tau \searrow 0} \frac{1}{\tau} \int_{-\tau}^{0} \chi^{\prime}\left(t_{3} / \tau\right) d t_{3}\right) \int_{Q} \tilde{\psi}(\bar{t}, 0) f(\omega(\bar{t})) \mathbf{e}_{3}(\bar{t}) \otimes \mathbf{e}_{3}(\bar{t}) g^{1 / 2}(\bar{t}) d t_{1} d t_{2}= \\
& \int_{Q} \tilde{\psi}(\bar{t}, 0) f(\omega(\bar{t})) \mathbf{e}_{3}(\bar{t}) \otimes \mathbf{e}_{3}(\bar{t}) g^{1 / 2}(\bar{t}) d t_{1} d t_{2}=\int_{\mathcal{S}} \psi(\omega) f(\omega) \mathbf{n}(\omega) \otimes \mathbf{n}(\omega) d s,
\end{aligned}
$$

which yields the first equality in (4.1). The second obviously follows from the first. $\square$.

Next lemma establishes the limiting relation for the integral involved the derivatives of the mapping $\mathbf{D}_{\tau}$ 
Lemma 4.2. Let the vector fields $\mathbf{h}, \mathbf{u}: \Omega \mapsto \mathbb{R}^{3}$ belongs to the class $C^{1}(\Omega)^{3}$ and vanishes on $\mathcal{S}$. Then

$$
\lim _{\tau \searrow 0} \int_{\Omega} \partial_{x_{i}} \mathbf{h}(x) \cdot\left(\partial_{x_{i}} \mathbf{D}\right) \mathbf{u} d x=-\int_{\mathcal{S}} f(\omega) \partial_{n} \mathbf{h}(\omega) \cdot(\mathbf{I}-\mathbf{n} \otimes \mathbf{n}) \partial_{n} \mathbf{u}(\omega) d s,
$$

Proof. Using the partition of unit we can assume that spt $\mathbf{h}$ is concentrated in the neighborhood of point $\omega_{0} \in \mathcal{S}$, which gives

$$
\int_{\Omega} \partial_{x_{i}} \mathbf{h}(x) \cdot\left(\partial_{x_{i}} \mathbf{D}\right) \mathbf{u} d x=\int_{Q} \int_{-\tau}^{0} \partial_{x_{i}} \mathbf{h}(X(\mathbf{t})) \cdot\left[\partial_{x_{i}} \mathbf{D}(X(\mathbf{t}))\right] \mathbf{u}(X(\mathbf{t})) \operatorname{det} X^{\prime}(\mathbf{t}) d \mathbf{t} .
$$

Notice that

$$
\partial_{x_{i}} \mathbf{h}(X(\mathbf{t})) \cdot\left[\partial_{x_{i}} \mathbf{D}(X(\mathbf{t}))\right] \mathbf{u}(X(\mathbf{t}))=G^{p q} \partial_{t_{p}} \tilde{\mathbf{h}}(\mathbf{t}) \cdot\left[\partial_{t_{q}} \mathbf{D}(X(\mathbf{t}))\right] \tilde{\mathbf{u}}(\mathbf{t})
$$

where

$$
\tilde{\mathbf{h}}(\mathbf{t})=\mathbf{h}(X(\mathbf{t})), \quad \tilde{\mathbf{u}}(\mathbf{t})=\mathbf{u}(X(\mathbf{t})), \quad G^{p q}=\left(\nabla_{X} t_{p}\right)(X(\mathbf{t})) \cdot\left(\nabla_{X} t_{q}\right)(X(\mathbf{t})) .
$$

Thus we get

$$
\int_{\Omega} \partial_{x_{i}} \mathbf{h}(x) \cdot\left(\partial_{x_{i}} \mathbf{D}\right) \mathbf{u} d x=\int_{Q} \int_{-\tau}^{0} G^{p q} \partial_{t_{p}} \tilde{\mathbf{h}}(\mathbf{t}) \cdot\left[\partial_{t_{q}} \mathbf{D}(X(\mathbf{t}))\right] \tilde{\mathbf{u}}(\mathbf{t}) \operatorname{det} X^{\prime}(\mathbf{t}) d \mathbf{t} .
$$

Since $\mathbf{u}$ vanishes at $\mathcal{S}$, the Taylor formula gives

$$
\tilde{\mathbf{u}}(\mathbf{t}) \equiv \mathbf{u}\left(\omega(\bar{t})+t_{3} \mathbf{n}(\omega(\bar{t}))=\partial_{n} \mathbf{u}(\omega(\bar{t})) t_{3}+r\left(\omega, t_{3}\right)\right.
$$

where

$$
\frac{1}{t_{3}} r\left(\omega, t_{3}\right) \rightarrow 0 \text { as } t_{3} \rightarrow 0 \text { uniformly with respect to } \omega \in \mathcal{S}
$$

Since the first derivatives of $\tilde{\mathbf{h}}$ and functions $G^{p q}$ are uniformly bounded and $\left|\partial_{t_{q}} \mathbf{D}\right| \leq c \tau^{-2}$ we have

$$
\int_{Q} \int_{-\tau}^{0}\left|G^{p q} \partial_{t_{p}} \tilde{\mathbf{h}}(\mathbf{t}) \cdot \partial_{t_{q}} \mathbf{D}(X(\mathbf{t})) r(\mathbf{t})\right| \operatorname{det} X^{\prime}(\mathbf{t}) d \mathbf{t} \leq c \tau^{-2} \int_{Q} \int_{-\tau}^{0}|r(\mathbf{t})| d \mathbf{t} \rightarrow 0
$$

as $\tau \rightarrow 0$. Thus we get

$$
\begin{aligned}
& \lim _{\tau \searrow 0} \int_{\Omega} \partial_{x_{i}} \mathbf{h}(x) \cdot \partial_{x_{i}} \mathbf{D} \mathbf{u} d x= \\
& \lim _{\tau \searrow 0} \int_{Q} \int_{-\tau}^{0} t_{3} G^{p q} \partial_{t_{p}} \tilde{\mathbf{h}}(\mathbf{t}) \cdot \partial_{t_{q}} \mathbf{D}(X(\mathbf{t})) \partial_{n} \mathbf{u}(\omega(\bar{t})) \operatorname{det} X^{\prime}(\mathbf{t}) d t_{1} d t_{2} d t_{3}
\end{aligned}
$$

Next it follows from the representation (3.12) that

$$
\left|\partial_{t_{q}} \mathbf{D}_{\tau}\right| \leq c \tau^{-1} \text { for } q=1,2
$$

and

$$
\partial_{t_{3}} \mathbf{D}_{\tau}=\frac{1}{\tau^{2}} \chi^{\prime \prime}\left(t_{3} / \tau\right) f(\omega(\bar{t}))(\mathbf{I}-\mathbf{n} \otimes \mathbf{n})+R_{1} \text { with }\left|R_{1}(\mathbf{t})\right| \leq c \tau^{-1}
$$


It follows from this that

$$
\begin{aligned}
& t_{3} G^{p q} \partial_{t_{p}} \tilde{\mathbf{h}}(\mathbf{t}) \cdot\left[\partial_{t_{q}} \mathbf{D}(X(\mathbf{t}))\right] \partial_{n} \mathbf{u}(\omega(\bar{t})) \operatorname{det} X^{\prime}(\mathbf{t})= \\
& \frac{t_{3}}{\tau^{2}} \chi^{\prime \prime}\left(t_{3} / \tau\right) f(\omega(\bar{t})) G^{p 3} \partial_{t_{p}} \tilde{\mathbf{h}}(\mathbf{t}) \cdot(\mathbf{I}-\mathbf{n} \otimes \mathbf{n}) \partial_{n} \mathbf{u}(\omega(\bar{t})) \operatorname{det} X^{\prime}(\mathbf{t})+R_{2},
\end{aligned}
$$

where

$$
\left|R_{2}\right| \leq c\left|t_{3}\right| / \tau \leq c
$$

Using formula (3.4) for the Jacobian we obtain

$$
\begin{aligned}
& t_{3} G^{p q} \partial_{t_{p}} \tilde{\mathbf{h}}(\mathbf{t}) \cdot\left[\partial_{t_{q}} \mathbf{D}(X(\mathbf{t}))\right] \partial_{n} \mathbf{u}(\omega(\bar{t})) \operatorname{det} X^{\prime}(\mathbf{t})= \\
& \frac{t_{3}}{\tau^{2}} \chi^{\prime \prime}\left(t_{3} / \tau\right) f(\omega(\bar{t})) G^{p 3} \partial_{t_{p}} \tilde{\mathbf{h}}(\mathbf{t}) \cdot(\mathbf{I}-\mathbf{n} \otimes \mathbf{n}) \partial_{n} \mathbf{u}(\omega(\bar{t})) g^{1 / 2}+R_{3},
\end{aligned}
$$

where $\left|R_{3}\right| \leq c$. From this we conclude that

$$
\begin{aligned}
& \lim _{\tau \searrow 0} \int_{\Omega} \partial_{x_{i}} \mathbf{h}(x) \cdot\left(\partial_{x_{i}} \mathbf{D}\right) \mathbf{u} d x= \\
& \lim _{\tau \searrow 0} \frac{1}{\tau^{2}} \int_{Q} \int_{-\tau}^{0} t_{3} \chi^{\prime \prime}\left(t_{3} / \tau\right) f(\omega(\bar{t})) G^{p 3} \partial_{t_{p}} \tilde{\mathbf{h}}(\mathbf{t}) \cdot(\mathbf{I}-\mathbf{n} \otimes \mathbf{n}) \partial_{n} \mathbf{u}(\omega(\bar{t})) g^{1 / 2} d \mathbf{t}
\end{aligned}
$$

Since the vector field $\tilde{\mathbf{h}}$ vanishes at $t_{3}=0$ we have for $\alpha=1,2$

$$
\partial_{t_{\alpha}} \tilde{\mathbf{h}}=r_{\alpha}(\mathbf{t}), \partial_{t_{3}} \tilde{\mathbf{h}}(\mathbf{t})-\partial_{t_{3}} \tilde{\mathbf{h}}(\bar{t}, 0)=r_{3}(\mathbf{t}),
$$

where $r_{i}(\mathbf{t}) \rightarrow 0$ as $t_{3} \rightarrow 0$ uniformly in $\bar{t} \in Q$. In particular we have

$$
\begin{array}{r}
\frac{1}{\tau^{2}}\left|\int_{Q} \int_{-\tau}^{0} t_{3} \chi^{\prime \prime}\left(t_{3} / \tau\right) f(\omega(\bar{t})) G^{p 3} r_{p}(\mathbf{t}) \cdot(\mathbf{I}-\mathbf{n} \otimes \mathbf{n}) \partial_{n} \mathbf{u}(\omega(\bar{t})) g^{1 / 2} d \mathbf{t}\right| \leq \\
\frac{c}{\tau^{2}} \int_{Q} \int_{-\tau}^{0}\left|t_{3}\right|\left(\left|r_{1}\right|+\left|r_{2}\right|+\left|r_{3}\right|\right) d \mathbf{t} \rightarrow 0 \text { as } \tau \rightarrow 0 .
\end{array}
$$

Combining obtained results we arrive at the equality

$$
\begin{aligned}
& \lim _{\tau \searrow 0} \int_{\Omega} \partial_{x_{i}} \mathbf{h}(x) \cdot \partial_{x_{i}} \mathbf{D} \mathbf{u} d x= \\
& \lim _{\tau \searrow 0} \frac{1}{\tau^{2}} \int_{Q} \int_{-\tau}^{0} t_{3} \chi^{\prime \prime}\left(t_{3} / \tau\right) f(\omega(\bar{t})) G^{33} \partial_{t_{3}} \tilde{\mathbf{h}}(\bar{t}, 0) \cdot(\mathbf{I}-\mathbf{n} \otimes \mathbf{n}) \partial_{n} \mathbf{u}(\omega(\bar{t})) g^{1 / 2} d \mathbf{t}
\end{aligned}
$$

Recalling that

$$
\partial_{t_{3}} \tilde{\mathbf{h}}(\bar{t}, 0)=\partial_{n} \mathbf{h}(\omega(\bar{t})) \text { and } G^{33}=\mathbf{e}_{3} \cdot \mathbf{e}_{3}=1
$$

and using the identity

$$
\frac{1}{\tau^{2}} \int_{-\tau}^{0} t_{3} \chi^{\prime \prime}\left(t_{3} / \tau\right) d t_{3}=-1
$$


we finally obtain

$$
\begin{aligned}
& \lim _{\tau \searrow 0} \int_{\Omega} \partial_{x_{i}} \mathbf{h}(x) \cdot\left(\partial_{x_{i}} \mathbf{D}\right) \mathbf{u} d x= \\
& \lim _{\tau \searrow 0} \frac{1}{\tau^{2}} \int_{Q} \int_{-\tau}^{0} t_{3} \chi^{\prime \prime}\left(t_{3} / \tau\right) f(\omega(\bar{t})) \partial_{n} \mathbf{h}(\omega(\bar{t})) \cdot(\mathbf{I}-\mathbf{n} \otimes \mathbf{n}) \partial_{n} \mathbf{u}(\omega(\bar{t})) g^{1 / 2} d \mathbf{t}= \\
& \left(\lim _{\tau \searrow 0} \frac{1}{\tau^{2}} \int_{-\tau}^{0} t_{3} \chi^{\prime \prime}\left(t_{3} / \tau\right) d t\right) \int_{Q} f(\omega(\bar{t})) \partial_{n} \mathbf{h}(\omega(\bar{t})) \cdot(\mathbf{I}-\mathbf{n} \otimes \mathbf{n}) \partial_{n} \mathbf{u}(\omega(\bar{t})) g^{1 / 2} d t_{1} d t_{2}= \\
& -\int_{Q} f(\omega(\bar{t})) \partial_{n} \mathbf{h}(\omega(\bar{t})) \cdot(\mathbf{I}-\mathbf{n} \otimes \mathbf{n}) \partial_{n} \mathbf{u}(\omega(\bar{t})) g^{1 / 2} d t_{1} d t_{2}= \\
& -\int_{\mathcal{S}} f(\omega) \partial_{n} \mathbf{h}(\omega) \cdot(\mathbf{I}-\mathbf{n} \otimes \mathbf{n}) \partial_{n} \mathbf{u}(\omega) d s
\end{aligned}
$$

which yields (4.2) and the lemma follows.

\section{Proof of Theorem 1.3}

Fix an arbitrary function $\eta \in C^{\infty}(\Omega)$ satisfying the boundary conditions $\eta=0$ on $\Sigma$ so that $\eta=1$ in a neighborhood of $\mathcal{S}$. Next choose the perturbation $\mathbf{T}_{\tau}$ in the form (3.9). Notice that the derivatives of the function $\eta$ vanish in a neighborhood of $\mathcal{S}$ and the vector field $\mathbf{u}$ belongs to $C^{1}(\Omega)$. Substituting $\mathbf{T}_{\tau}$ into (1.21) integration by parts and applying Lemma 4.1 we obtain

$$
\lim _{\tau \searrow 0} L_{e}\left(\mathbf{T}_{\tau}\right)=0
$$

Since the function $\eta$ is infinitely differentiable the right hand sides $(A, B, C)$ of adjoint state equations $(1.25)$ belongs to $W^{1+s, r}(\Omega) \times \Pi W^{s, r}(\Omega) \times W^{s, r}(\Omega)$, it follows from Proposition 2.4 that $\left(h^{*}, g^{*}, \varsigma^{*}, v^{*}, l^{*}\right) \in W^{1+s, r}(\Omega) \times \Pi W^{s, r}(\Omega) \times W^{s, r}(\Omega) \times$ $\mathbb{R}$. Applying Proposition 2.4 to problem (1.29) we conclude that $(h, g, \varsigma, v, l) \in$ $W^{1+s, r}(\Omega) \times \Pi W^{s, r}(\Omega) \times W^{s, r}(\Omega) \times \mathbb{R}$.

It follows from this that the functions $g^{*}, \varsigma^{*}$, and $v^{*}$ are continuous. From this and Lemma 4.1 we obtain

$$
\lim _{\tau \searrow 0} \int_{\Omega} \operatorname{Tr} \mathbf{T}_{\tau}^{\prime}\left(b_{10} \varsigma^{*}+b_{20} g^{*}+\sigma v^{*}+l^{*} b_{30}\right) d x=\int_{\mathcal{S}} f(\omega)\left(b_{10} \varsigma^{*}+b_{20} g^{*}+\sigma v^{*}+l^{*} b_{30}\right) d s .
$$

Next we have

$$
\begin{array}{r}
\int_{\Omega} \mathscr{D}\left(\mathbf{T}_{\tau}\right) \cdot \mathbf{h}^{*} d x=\int_{\Omega} \operatorname{div}\left[\left(\operatorname{Tr} \mathbf{T}_{\tau}^{\prime} \mathbf{I}-\mathbf{D}_{\tau}-\mathbf{D}_{\tau}^{*}\right) \nabla u\right] \cdot h^{*} d x+ \\
\int_{\Omega}\left(D_{\tau}^{*} \Delta \mathbf{u}+\Delta\left(\mathbf{D}_{\tau} \mathbf{u}\right)\right) \cdot \mathbf{h}^{*} d x- \\
R \int_{\Omega} \varrho\left[\mathbf{D}_{\tau}^{*}(\mathbf{u} \nabla \mathbf{u})+\mathbf{u} \nabla\left(\mathbf{D}_{\tau} \mathbf{u}\right)\right] \cdot \mathbf{h}^{*} d x=I_{1}(\tau)+I_{2}(\tau)+I_{3}(\tau) .
\end{array}
$$


Recall that by virtue of definition of the spaces $Y^{s, r}$, we have $\mathbf{u} \in Y^{s, r} \subset W^{2,2}(\Omega)$ and $\varrho \in X^{s, r} \subset W^{1,2}(\Omega)$ On the other hand, the vector field $\mathbf{h}^{*}$ has continuous derivatives in $\Omega$. Hence we can integrate by parts to obtain the identity

$$
I_{3}(\tau)=R \int_{\Omega}\left[\varrho \mathbf{D}_{\tau}^{*}(\mathbf{u} \nabla \mathbf{u}) \cdot \mathbf{h}^{*}-\varrho\left(\mathbf{u} \nabla \mathbf{h}^{*}\right) \cdot\left(\mathbf{D}_{\tau} \mathbf{u}\right)\right] d x
$$

Here we use the equality $\operatorname{div}(\varrho \mathbf{u})=0$. Since $\varrho, \nabla \mathbf{u}, \nabla \mathbf{h}^{*}$ are continuous and $\mathbf{u}, \mathbf{h}^{*}$ vanishes at $\mathcal{S}$, we can apply Lemma 4.1 to obtain $\lim _{\tau \backslash 0} I_{3}(\tau)=0$. Next introduce the matrix

$$
V=\operatorname{Tr} \mathbf{T}_{\tau}^{\prime} \mathbf{I}-\mathbf{D}_{\tau}-\mathbf{D}_{\tau}^{*}
$$

Note that

$$
(V \nabla \mathbf{u})_{i j}=V_{i k} \partial_{x_{k}} u_{j}, \quad[\operatorname{div}(V \nabla \mathbf{u})]_{j}=\partial_{x_{i}}\left(V_{i k} \partial_{x_{k}} u_{j}\right)
$$

Thus we get

$$
I_{1}(\tau) \equiv \int_{\Omega} \operatorname{div}(V \nabla \mathbf{u}) \cdot \mathbf{h}^{*}=-\int_{\Omega} V_{i k} \partial_{x_{k}} u_{j} \partial_{x_{i}} h_{j}^{*} d x
$$

It follows from Lemma 4.1 and the equality $\mathbf{D}=\operatorname{Tr} \mathbf{T I}-\mathbf{T}^{\prime}$ that for any $\psi \in C(\Omega)$,

$$
\lim _{\tau \searrow 0} \int_{\Omega} V_{i k} \psi d x=\int_{\mathcal{S}} f(\omega)\left(-\delta_{i k}+2 n_{i} n_{k}\right) \psi(\omega) d s
$$

Since $\nabla \mathbf{u}$ and $\nabla \mathbf{h}^{*}$ are continuous, we conclude from this that

$$
\lim _{\tau \searrow 0} I_{1}(\tau)=\int_{\mathcal{S}} f(\omega) \partial_{x_{i}} u_{j} \partial_{x_{i}} h_{j}^{*} d s-2 \int_{\mathcal{S}} f(\omega) n_{k} \partial_{x_{k}} u_{j} n_{i} \partial_{x_{i}} h_{j}^{*} d s
$$

Recalling that $\mathbf{u}$ and $\mathbf{h}^{*}$ vanish on $\partial f$ we obtain

$$
\partial_{x_{i}} u_{j} \partial_{x_{i}} h_{j}^{*}=\partial_{n} \mathbf{u} \cdot \partial_{n} \mathbf{h}^{*}, \quad n_{k} \partial_{x_{k}} u_{j} n_{i} \partial x_{i} h_{j}^{*}=\partial_{n} \mathbf{u} \cdot \partial_{n} \mathbf{h}^{*},
$$

which gives

$$
\lim _{\tau \searrow 0} I_{1}(\tau)=-\int_{\mathcal{S}} f(\omega) \partial_{n} \mathbf{u} \cdot \partial_{n} \mathbf{h}^{*} d s
$$

Next integrating by parts we obtain

$$
I_{2}(\tau)=-\int_{\Omega}\left[\left(\mathbf{D}_{\tau}+\mathbf{D}_{\tau}^{*}\right) \partial_{x_{i}} \mathbf{u} \cdot \partial_{x_{i}} \mathbf{h}^{*}+\partial_{x_{i}} \mathbf{u} \cdot\left(\partial_{x_{i}} \mathbf{D}_{\tau}\right) \mathbf{h}^{*}+\partial_{x_{i}} \mathbf{h}^{*} \cdot\left(\partial_{x_{i}} \mathbf{D}_{\tau}\right) \mathbf{u}\right] d x
$$

Applying Lemma 4.1 and 4.2 we arrive at the equality

$$
\begin{array}{r}
\lim _{\tau \searrow 0} I_{2}(\tau)=-2 \int_{\mathcal{S}} f(\omega)\left[\partial_{x_{i}} \mathbf{u} \cdot \partial_{x_{i}} \mathbf{h}^{*}-\left(\mathbf{n} \cdot \partial_{x_{i}} \mathbf{u}\right)\left(\mathbf{n} \cdot \partial_{x_{i}} \mathbf{h}^{*}\right)\right] d s+ \\
2 \int_{\mathcal{S}} f(\omega)\left[\partial_{n} \mathbf{u} \cdot \partial_{n} \mathbf{h}^{*}-\left(\mathbf{n} \cdot \partial_{n} \mathbf{u}\right)\left(\mathbf{n} \cdot \partial_{n} \mathbf{h}^{*}\right)\right] d s
\end{array}
$$

Since $\mathbf{u}$ and $\mathbf{h}^{*}$ vanish on $\mathcal{S}$, we have $\partial_{x_{i}} \mathbf{u}=n_{i} \partial_{n} \mathbf{u}$ and $\partial_{x_{i}} \mathbf{h}^{*}=n_{i} \partial_{n} \mathbf{h}^{*}$ which gives

$$
\partial_{x_{i}} \mathbf{u} \cdot \partial_{x_{i}} \mathbf{h}^{*}-\left(\mathbf{n} \cdot \partial_{x_{i}} \mathbf{u}\right)\left(\mathbf{n} \cdot \partial_{x_{i}} \mathbf{h}^{*}\right)=\partial_{n} \mathbf{u} \cdot \partial_{n} \mathbf{h}^{*}-\left(\mathbf{n} \cdot \partial_{n} \mathbf{u}\right)\left(\mathbf{n} \cdot \partial_{n} \mathbf{h}^{*}\right) .
$$


Thus we get $\lim _{\tau \searrow 0} I_{2}(\tau)=0$. Combining (1.20) and (1.26) we arrive at the following expression for the shape derivative of the drag functional

$$
\left.\frac{d}{d \varepsilon} J_{D}\left(\mathfrak{S}_{\varepsilon}\right)\right|_{\varepsilon=0}=L_{e}\left(\mathbf{T}_{\tau}\right)+\int_{\Omega} \operatorname{Tr} \mathbf{T}_{\tau}^{\prime}\left(b_{10} \varsigma^{*}+b_{20} g^{*}+\sigma v^{*}+l^{*} b_{30}\right) d x+I_{1}(\tau)+I_{2}(\tau)+I_{3}(\tau) .
$$

Letting $\tau \searrow 0$ we finally obtain

$$
\left.\frac{d}{d \varepsilon} J_{D}\left(\mathfrak{S}_{\varepsilon}\right)\right|_{\varepsilon=0}=\int_{\mathcal{S}} f(\omega)\left(b_{10} \varsigma^{*}+b_{20} g^{*}+\sigma v^{*}+l^{*} b_{30}-\partial_{n} \mathbf{u} \cdot \partial_{n} \mathbf{h}^{*}\right) d s .
$$

It remains to note that by virtue of identity (1.30),

$$
\left(g^{*}, \varsigma^{*}, v^{*}, l^{*}\right)=(g, \varsigma, v, l), \quad \partial_{n} \mathbf{h}^{*}=\partial_{n} \mathbf{h} \text { on } \mathcal{S},
$$

and the theorem follows.

\section{REFERENCES}

[1] R.A, Adams Sobolev spaces Academic press, New-York (1975)

[2] V. Barbu, I. Lasiecka, R. Triggiani Tangential boundary stabilization of Navier-Stokes equations Memoirs of the AMS, 181(2006), Providence, 128pp.

[3] J. A. Bello, E. Fernandez-Cara, J. Lemoine, J. Simon The differentiability of the drag with respect to variations of a Lipschitz domain in a Navier-Stokes flow, SIAM J. Control. Optim. 35, No. 2, (1997), 626-640.

[4] J.A. Bello, E. Fernandez-Cara, and J. Simon Optimal shape design for Navier-Stokes flow, in System Modelling and Optimization, Lecture Notes in Control and Inform Sci 180, D. Kall, ed., Springer-Verlag, Berlin, (1998).

[5] J. Bergh, J. Löfström, Interpolation spaces. An Introduction, Springer-Verlag, Berlin Heidelberg New-York (1976)

[6] M.C. Delfour, J.-P. Zolesio Shapes and Geometries, Advances in Design and Control. Society for Industrial and Applied Mathematics (SIAM), Philadelphia, PA, 2001.

[7] G. Galdi An introduction to the mathematical theory of the Navier-Stokes equations V.I, Springer-Verlag, Berlin, Heidelberg New-York,(1998)

[8] P.Grisward Caracterisation de Quelques Espaces, d' Interpolation Arch. Rat. Mech. Anal. 25 (1967), 43-63

[9] E. Feireisl, A.H. Novotný, H.Petzeltová On the domain dependence of solutions to the compressible Navier-Stokes equations of a barotropic fluid Math. Methods Appl. Sci. 25 (2002), no. 12, 1045-1073.

[10] E. Feireisl Shape optimization in viscous compressible fluids Appl. Math. Optim. 47(2003), 59-78.

[11] A. Fursikov Optimal control of distributed systems: theory and applications Translation of Mathematical monographs v.187, AMS, Providence, Rhode Island, 2000.

[12] B. Kawohl, O. Pironneau, L. Tartar and J. Zolesio Optimal Shape Design Lecture Notes in Math. 1740, Springer-Verlag, 2000.

[13] J. Löfström Interpolation of boundary value problems of Neumann type on smooth domains J. London Math. Soc. 46 (1992), 499-516.

[14] J. Löfström Interpolation of subspaces, Techn. Rep. N 10, University of Göterborg (1997) 63pp.

[15] B. Mohammadi, O. Pironneau Shape optimization in fluid mechanics Ann. Rev. Fluid Mech. $36,255-279,(2004)$.

[16] A. Novotný, M. Padula Existence and Uniqueness of Stationary solutions for viscous compressible heat conductive fluid with large potential and small non-potential external forces Siberian Math. Journal, 34, 1993, 120-146

[17] A. Novotný, I. Straškraba Introduction to the mathematical theory of compressible flow Oxford Lecture Series in Mathematics and its Applications, Vol. 27. Oxford University Press, Oxford, 2004. 
[18] M. Padula Existence and uniqueness for viscous steady compressible motions Arch. Rational Mech. Anal., 97, no 1, 1-20 (1986).

[19] P.I. Plotnikov, J. Sokolowski Domain dependence of solutions to compressible Navier-Stokes equations SIAM J. Control Optim., Volume 45, Issue 4, 2006, pp. 1147-1539.

[20] P.I. Plotnikov, E.V. Ruban, J. Sokolowski Inhomogeneous boundary value problems for compressible Navier-Stokes equations, well-posedness and sensitivity analysis, SIAM J. Math. Analysis 40 (2008), 1152-1200.

[21] P.I. Plotnikov, E.V. Ruban, J. Sokolowski Inhomogeneous boundary value problems for compressible Navier-Stokes and transport equations, Journal des Mathématiques Pure et Appliquées, electronic. (2009).

[22] J. Simon Domain variation for drag in Stokes flow, in Control and Estimation of Distributed Parameter Systems, Internat. N Ser. Numer. Math. 91, F. Kappel, K. Kuninisch, and W. Schappacher, eds., Birkhäuser, Basel, 1989, 361-378.

[23] T. Slawig A formula for the derivative with respect to domain variations in Navier-Stokes flow based on an embedding domain method SIAM J. Control Optim., 42, No 2, 495-512 (2003).

[24] T. Slawig An explicit formula for the derivative of a class of cost functionals with respect to domain variations in Stokes flow SIAM J. Control Optim., 39, No 1, 141-158 (2000).

[25] L. Tartar An introduction in Sobolev spaces and interpolation spaces. Lecture notes of the Unione Matematica Italiana 3, Springer, Berlin; UMI, Bologna (2007).

[26] J. Sokolowski, J.-P. Zolésio Introduction to Shape Optimization. Shape Sensitivity Analysis. Springer Series in Computational Mathematics Vol. 16, Springer Verlag, (1992).

Lavryentyev Institute of Hydrodynamics, Siberian Division of Russian Academy of Sciences, Lavryentyev Pr. 15, Novosibirsk 630090, Russia

E-mail address: plotnikov@hydro.nsc.ru

Institut Elie Cartan, UMR 7502 NanCy-Université-CNRS-INRIA, Laboratoire de Mathématiques, Université Henri Poincaré Nancy 1, B.P. 239, 54506 Vandoeuvre lés Nancy Cedex, FRANCE

E-mail address: Jan.Sokolowski@iecn.u-nancy.fr

URL: http://www.iecn.u-nancy.fr/ sokolows/ 